\title{
Analysis of the Kondo effect in ferromagnetic atomic-sized contacts
}

\author{
M. R. Calvo, ${ }^{1,2}$ D. Jacob, ${ }^{3}$ and C. Untiedt ${ }^{1}$ \\ ${ }^{1}$ Departamento de Física Aplicada, Universidad de Alicante, Campus de San Vicente del Raspeig, E-03690 Alicante, Spain \\ ${ }^{2}$ London Centre for Nanotechnology, University College London, 17-19 Gordon Street, London WC1H0AH, United Kingdom \\ ${ }^{3}$ Max-Planck-Institut für Mikrostrukturphysik, Weinberg 2, 06120 Halle, Germany
}

(Received 4 May 2012; published 20 August 2012)

\begin{abstract}
Atomic contacts made of ferromagnetic metals present zero-bias anomalies in the differential conductance due to the Kondo effect. These systems provide a unique opportunity to perform a statistical analysis of the Kondo parameters in nanostructures since a large number of contacts can be easily fabricated using break-junction techniques. The details of the atomic structure differ from one contact to another so a large number of different configurations can be statistically analyzed. Here we present such a statistical analysis of the Kondo effect in atomic contacts made from the ferromagnetic transition metals $\mathrm{Ni}, \mathrm{Co}$, and $\mathrm{Fe}$. Our analysis shows clear differences between materials that can be understood by fundamental theoretical considerations. This combination of experiments and theory allows us to extract information about the origin and nature of the Kondo effect in these systems and to explore the influence of geometry and valence in the Kondo screening of atomic-sized nanostructures.
\end{abstract}

DOI: 10.1103/PhysRevB.86.075447

PACS number(s): 73.63.Rt, 72.15.Qm, 75.75.-c

\section{INTRODUCTION}

The Kondo effect is one of the most intriguing phenomena arising from electronic correlations and was first observed over 80 years ago as a then-unexpected increase of the resistance of gold wires at very low temperatures. ${ }^{1}$ This phenomenon was successfully explained by Kondo 30 years later in his seminal work ${ }^{2}$ as due to scattering of conduction electrons off magnetic impurities present in the Au samples, thereby screening its magnetic moment. More generally, whenever a local magnetic moment is coupled to a sea of conduction electrons, the Kondo effect can arise at low-enough temperatures with important consequences for the electronic and magnetic properties of the system.

Also in the case of mesoscopic devices, the Kondo effect strongly alters the electronic structure and, therefore, has dramatic consequences on the transport characteristics of the system. One of the simplest mesoscopic devices showing the Kondo effect is the case of a quantum dot connected in series to two metallic leads ${ }^{3,4}$ : When an electronic level of a quantum dot is well below the Fermi energy of the metallic leads and the Coulomb repulsion is strong enough to prevent double occupation, the quantum dot behaves as a magnetic impurity. In this situation conduction through the quantum dot is usually strongly suppressed due to the Coulomb blockade. ${ }^{5}$ However, at low temperatures the Kondo effect restores the conductance due to the apparition of a sharp resonance-the so-called Kondo resonance-in the spectral function of the quantum dot right at the Fermi energy of the electrodes.

The essence of the Kondo effect is the formation of a total spin-singlet state between the impurity electrons and the conduction electrons near the Fermi level ${ }^{6}$ below a certain critical temperature characteristic of the system, the Kondo temperature. The formation of this Kondo singlet state gives rise to the effective screening of the magnetic moment of the impurity and leads to the formation of a sharp resonance in the spectral density of the impurity electrons right at the Fermi level. This is the aforementioned Kondo resonance, sometimes also called Abrikosov-Suhl resonance. ${ }^{7-9}$ In the case of magnetic impurities in metallic host materials, the formation of the Kondo resonance in the spectral density of the impurity leads to additional scattering of the conduction electrons, resulting in the increase of the resistance of the metal at low temperatures (for a review of the Kondo effect in bulk metals with magnetic impurities; see, e.g., Ref. 6).

Other systems where evidence for the Kondo effect has been found by the manifestation of a Kondo resonance, either in the spectral density or the conductance characteristics, include point contacts, ${ }^{10,11}$ different molecules containing magnetic atoms on surfaces, ${ }^{12,13}$ fullerenes, ${ }^{14}$ carbon nanotubes contacted by metallic electrodes, ${ }^{15,16}$ and magnetic atoms on surfaces studied by scanning tunneling microscopy (STM). ${ }^{17-25}$

In the case of magnetic adatoms on metal surfaces studied by STM, interference of different conduction channels through the atom (one of them bearing the Kondo resonance) gives rise to Fano line shapes ${ }^{26}$ in the low-bias conductance characteristics, similarly to the case of a quantum dot coupled laterally to a wire. ${ }^{27,28}$ By fitting those line shapes to the Fano model, one can obtain different parameters that describe the characteristics of the Kondo screening in the system, i.e., the width, position, and amplitude of the Kondo peak (for a review, see, e.g., Ref. 20). Of special relevance to our work are the STM experiments performed in the high-conductance regime when the tip is brought into contact with the adatom. ${ }^{29-37}$

In a recent work, we reported the observation of KondoFano line shapes in the conductance characteristics of atomic contacts made from ferromagnetic materials. ${ }^{38}$ In contrast to STM experiments where the contacted adatom can be imaged and the geometry of the system formed by the adatom and the surface can be completely characterized, break-junction experiments do not allow us to control the geometry of the system to the same extent (although they normally give rise to some geometrical repetition ${ }^{39}$ ). On the other hand, using break junctions, it is possible to form and study a large number of different configurations. ${ }^{40}$ 
The observation of the Kondo effect in ferromagnetic atomic contacts was highly unexpected for two reasons: First, given the chemical homogeneity of the atomic contacts, a devision into magnetic impurity and a spin-degenerate Fermi sea, as required for the appearance of the Kondo effect, is not obvious at all. Second, electrodes and the contact atoms are made from a ferromagnetic material. However, the Kondo effect and ferromagnetism are generally competing phenomena: For example, a strong-enough magnetic field corresponding to a Zeeman energy above the binding energy of the Kondo singlet (about the Kondo temperature) will break up the singlet and, thus, the Kondo effect, ${ }^{41}$ while on the other hand, magnetic fields on the order of the Kondo temperature or below will lead to a splitting of the Kondo resonance as shown, e.g., in Refs. 4,42, and 43.

Likewise, the Kondo resonance splits when a quantum dot is connected to ferromagnetic electrodes in the case of parallel alignment of the two electrodes' magnetic polarizations, while for antiparallel alignment of the electrodes, a normal Kondo effect is obtained. ${ }^{4-47}$ Therefore, one would expect that the coupling of the contact region to the strongly ferromagnetic bulk electrodes should either eliminate the Kondo effect completely or at least split the Kondo resonance unless the antiferromagnetic coupling between magnetic impurity and conduction electrons is strong enough compared to any other interactions.

Here, as in our previous work, ${ }^{38}$ we propose that the Kondo effect in ferromagnetic atomic contacts originates from individual $d$ levels of the undercoordinated tip atoms of the nanocontact, and the Kondo screening is due to the delocalized $s p$ electrons, which are basically spin unpolarized. Hence, the impurity or quantum dot bearing the spin consists of one or several $d$ levels of an individual contact atom.

In our model, depending on the material, the spin of the tip atom can be localized in different $d$ levels. Due to the low symmetry of the contact atoms, the individual $d$ levels couple differently to the $s p$-conduction electron bath, resulting in different Kondo screenings of the spin. This scenario may explain the different Kondo behavior observed for contacts made from $\mathrm{Fe}, \mathrm{Co}$, and $\mathrm{Ni}^{38}$ Contacts made of the same material may also present slightly different atomic configurations, which will also influence their Kondo properties. In our experiments, from the fitting of the differential conductance curves performed on atomic-sized contacts of ferromagnetic materials to a Fano expression, we extract the relevant parameters that characterize the Kondo effect on each contact.

In this work, the validity of the Fano-Kondo model for the contact regime, and, more specifically, for the case of atomic contacts, is revised and discussed. We present a more exhaustive analysis than in Ref. 38 for all the parameters of the Fano-Kondo model in our system and extract new information from the data, for instance, the distributions for the occupation of the $d$ level for Fe, Co, and $\mathrm{Ni}$. This further analysis confirms the marked differences of the case of $\mathrm{Ni}$ with respect to $\mathrm{Fe}$ and Co. We propose here a new interpretation of the data: The nature of the Kondo screening for $\mathrm{Ni}$ differs from that for $\mathrm{Fe}$ and Co. From the combined statistical analysis of the Kondo parameters and theoretical fundamental considerations, we can deduce the influence of valence and environment on the Kondo screening in nanostructures.
This paper is organized as follows: The first two sections are introductory. First, we review the transport properties of atomic contacts in Sec. II. In Sec. III, we then present the basic elements of the theory of the Kondo effect in the framework of the Anderson impurity model and justify the validity of the Fano-Kondo model for the case of atomic contacts of transition metals. Sections IV and V are devoted to the experimental methods and the presentation and discussion of the experimental results, respectively. We, finally, discuss the results in the framework of the theory presented in Sec. VI and summarize our main conclusions in Sec. VII.

\section{TRANSPORT IN ATOMIC CONTACTS}

The system under study in this work are atomic contacts, ${ }^{40}$ i.e., a contact between two metallic leads mediated by an atom forming a metallic bond with both leads. More specifically, we focus here on the case of homogeneous contacts, those where all the atoms forming the structure, leads, and contact are of the same element. The formation of an atomic contact can be identified from electronic transport measurements. When two pieces of the same pure metal are brought into contact, the conductance plotted against the inter-electrode distance shows a plateau when the atomic contact is formed, close to the quantum of conductance $G_{0}=2 e^{2} / h$. The exact value of this quantity will depend on the material and the atomic configuration of the contact. In general, the atomic orbitals will define a number of eigenchannels, each of these with a transmission probability $T_{i}$ which will be reflected in the total conductance through the Landauer formula $G=G_{0} \sum_{i} T_{i}$. The number of channels will be related to the valence of the metal $^{48,49}$ and will normally include a high transmitive $s$-type channel and several other with lower transmission, resulting in a final conductance in the range of $0.7-2.5 G_{0}$. Experimentally, the evolution of the conductance can be recorded over the formation or breaking process of the contact showing in most of the cases ${ }^{50}$ plateaus, not only for the one-atom contact but for every atomic rearrangement of the wire while pulling.

The one-atom plateau of conductance for the case of gold is near the quantum of conductance and, as shown in Fig. 1(a), higher for the case of the $3 d$ transition metals $\mathrm{Fe}, \mathrm{Co}$, and $\mathrm{Ni}$. From many of these traces we can build histograms which help us to identify the transport properties of the most probable atomic configurations of the contacts.

Different authors ${ }^{51,52}$ agree that, under cryogenic conditions and considering a high-enough amount of data (thousands of conductance traces), the conductance of monatomic contacts made from $\mathrm{Fe}, \mathrm{Co}$, or $\mathrm{Ni}$ takes a value higher than the quantum of conductance $G_{0}=2 e^{2} / h$, as expected in general for transition metals. ${ }^{40}$ Histograms of conductance of $\mathrm{Fe}$, $\mathrm{Co}$, and $\mathrm{Ni}$ constructed from thousands of breaking traces at 4.2 $\mathrm{K}$ are shown in Fig. 1(b). The most probable values for the conductance of the monatomic contacts are $1.2 G_{0}$, $1.6 G_{0}$, and $2 G_{0}$ for $\mathrm{Co}, \mathrm{Ni}$, and $\mathrm{Fe}$, respectively. Conductance values between $1 G_{0}$ and $2 G_{0}$ for ferromagnetic nanocontacts are in overall agreement with theoretical calculations. ${ }^{53-56}$ The conduction of these atomic contacts is the sum of the contributions of different channels, where the $s$ channel is expected to be open and practically degenerate in spin and, thus, to have an associated conductance of nearly $2 e^{2} / h$. In 


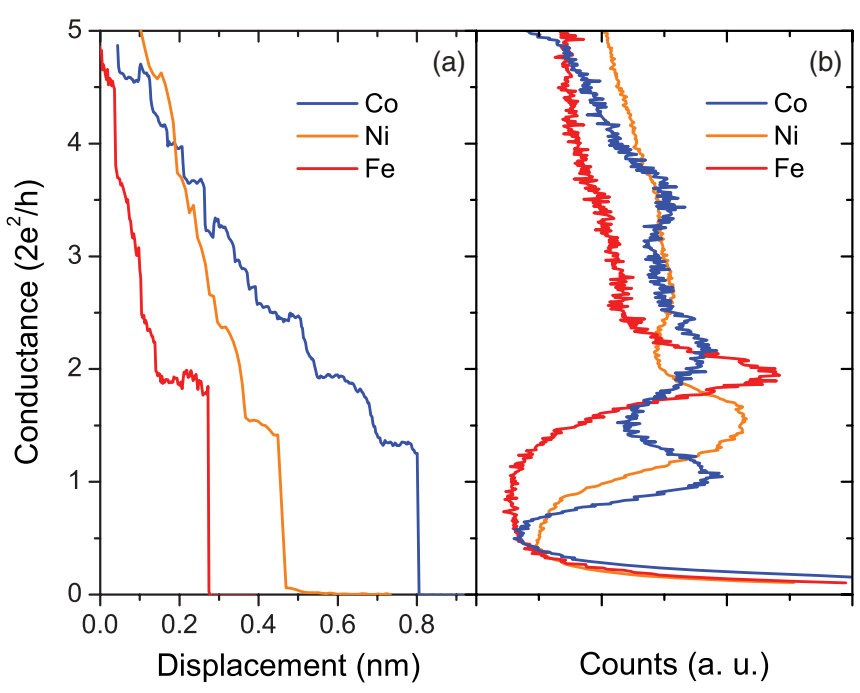

FIG. 1. (Color online) (a) Typical traces of conductance for breaking contacts of $\mathrm{Co}, \mathrm{Fe}$, and $\mathrm{Ni}$. (b) Histograms of conductance are constructed from thousands of these breaking traces of contacts fabricated by STM at $4.2 \mathrm{~K}$ for Ni, Fe, or Co. The histograms for the three materials show a clear first peak at values above $2 e^{2} / h$.

the ferromagnetic metals $\mathrm{Fe}, \mathrm{Co}$, and $\mathrm{Ni}$, another contribution to the overall conductance comes from the transmission of electrons via the five $3 d$ orbitals. However, due to the directionality and stronger localization of the $d$ orbitals, the electrons in the $d$ channels are easily scattered. Hence, the transmission in $d$ channels is usually far from perfect, as has been shown by ab initio calculations. ${ }^{53}$ In this work it was also shown that only the $d$ channels are spin polarized while the $s$ channel basically is unpolarized.

\section{BASIC ELEMENTS OF THE THEORY}

As discussed in the Introduction, in the experiments the presence of the Kondo effect is reflected in the conductance of a one-atom contact as a zero-bias anomaly. The details of this anomaly can be related to the characteristics of the Kondo effect in the framework of the Anderson impurity model (AIM). ${ }^{57}$ The purpose of this section is to provide the basic elements for a theoretical description of the Kondo effect in the framework of the single-level Anderson impurity model (1AIM) and the two-level Anderson impurity model (2AIM). This will allow us to properly analyze our experiments for different materials. Additionally, we develop a simple microscopic model in order to understand the occurrence of different Fano line shapes within the same material in terms of the variation of microscopic interactions due to variation in the atomic structure in the contact region.

\section{A. Model of a nanocontact}

We assume that the Kondo effect takes place in the under coordinated tip atoms of the nanocontact. This assumption seems reasonable considering that the Kondo effect is not observed in bulk samples of the ferromagnetic materials studied here. Hence, the Kondo effect must be related to the atomic-size constriction of the nanocontact. Furthermore, (a)
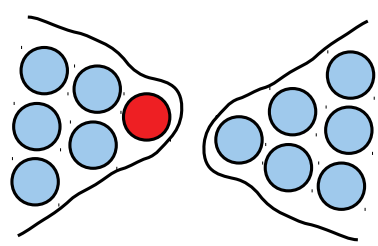

(b)

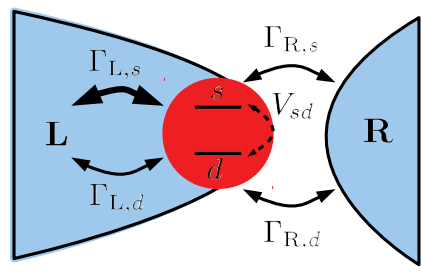

FIG. 2. (Color online) (a) Sketch of nanocontact just before breaking. The tip atom (red) of the left electrode is only weakly coupled to the right part of the nanocontact (and vice versa). This leads to the simplified model of the nanocontact as shown in (b). In the simplified model the tip atom (red) consists of a noninteracting $s$ level and a strongly interacting $d$ level which couple more strongly to the left electrode than to the right electrode $\left(\Gamma_{L, \alpha}>\Gamma_{R, \alpha}\right)$. The hybridization $V_{s d}$ between the $s$ and $d$ levels is due to crystal field splitting and is weak compared to the other energies. Moreover, the coupling of the $d$ level to the electrodes is much weaker than the coupling of the $s$ level $\left(\Gamma_{s} \gg \Gamma_{d}\right)$.

the Coulomb interaction and the localization of the electrons within the atomic-size constriction, and especially at the tip atoms, should be enhanced as compared to bulk.

Typically, the nanocontacts of the ferromagnetic metals Fe, $\mathrm{Co}$, and $\mathrm{Ni}$ form dimers in the last step before breaking, ${ }^{58,59}$ as in Fig. 2(a). In this case, each tip atom will be more strongly coupled to one electrode than to the other. As stated in Sec. II, the main conduction channel through the tip atoms is the spindegenerate $s$ channel with nearly perfect transmission. The $d$ channels, on the other hand, are strongly spin-polarized and their transmission is weaker or even completely blocked due to scattering by the geometry. ${ }^{53}$ As we have shown in our previous work ${ }^{38}$ by means of ab initio calculations, due to disorder and low coordination in the contact region one of the $d$ levels of the tip atom couples only very weakly to the $d$ levels of the neighboring atoms and instead couples only to the basically spin-degenerate $s$ channel. This is the situation where the Kondo effect can take place and which is described by the Anderson impurity model: A strongly interacting $d$ level couples to a noninteracting sea of conduction electrons. Hence, we can identify this $d$ level with the "quatum dot" or impurity in our experiments. This situation is schematically depicted in Fig. 2(b).

This scenario is further supported by recent dynamical mean-field theory calculations of a $\mathrm{Ni}$ nanocontact connected to electrodes made from $\mathrm{Cu}$ instead of $\mathrm{Ni}$, thus neglecting the ferromagnetic coupling to the bulk electrodes. ${ }^{60}$ In this situation, a Kondo effect emerges in one of the $d$ channels of the tip atoms of the Ni nanocontact with the Kondo temperature, in good agreement with the ones measured for Ni nanocontacts.

\section{B. Kondo effect in the Anderson model}

As we have argued in our previous work on the basis of $a b$ initio calculations, the combined effect of undercoordinated tip atoms and disorder in the contact region can lead to the selection of a single $d$ level of the tip atom that is only weakly coupled to the $d$ levels of the neighboring atoms. Hence, the magnetic coupling to the neighboring atoms is reduced for 
this level and, therefore, the Kondo effect can arise if this level is half-filled. This situation can be fulfilled in the case of $\mathrm{Ni}$ nanocontacts where the $d$ shell is $d^{9}$ and, thus, has a hole. In this case, the single-level Anderson impurity model (1AIM) where a single strongly interacting $d$ level is coupled to a bath of noninteracting conduction electrons is a good description of the situation,

$$
\begin{aligned}
\hat{\mathcal{H}}_{\mathrm{AIM}}= & \epsilon_{d} \hat{n}_{d}+U \hat{n}_{d \uparrow} \hat{n}_{d \downarrow}+\sum_{q, \sigma} \epsilon_{q} \hat{c}_{q \sigma}^{\dagger} \hat{c}_{q \sigma} \\
& +\sum_{q, \sigma}\left(V_{q} \hat{c}_{q \sigma}^{\dagger} \hat{d}_{\sigma}+V_{q}^{*} \hat{d}_{\sigma}^{\dagger} \hat{c}_{q \sigma}\right),
\end{aligned}
$$

where $\epsilon_{d}$ is the energy of the $d$ level, $U$ is the (effective) Coulomb repulsion between two electrons in the $d$ level, $\epsilon_{q}$ is the energy dispersion of the bath electrons, and $V_{q}$ is the coupling (or hopping) between the bath and the $d$ level. In the cases of $\mathrm{Fe}$ and $\mathrm{Co}$, there is more than one hole in the $d$ shell and, hence, one should consider a multilevel AIM. We will do so in the next subsection.

At zero temperature, the Anderson model is a Fermi liquid and, hence, has a (renormalized) quasiparticle resonance (i.e., the Kondo peak in the Kondo regime) near the Fermi level. The Green's function of the $d$ level is given by

$$
G_{d}(\omega)=\frac{z}{\omega-\epsilon_{K}+i \Gamma_{K}},
$$

where $z$ is the quasiparticle weight (i.e., the renormalization of the single-particle wave function due to many-body effects), $\epsilon_{K}$ is the position of the quasiparticle peak with respect to the Fermi level (set to zero for convenience), and $\Gamma_{K}=k T_{K}$ is half the width of the quasiparticle resonance which defines the Kondo temperature. Correspondingly, the projected density of state of the $d$ level is a Lorentzian centered at $\epsilon_{K}$ and width $2 \Gamma_{K}$,

$$
\rho_{d}(\omega)=-\frac{1}{\pi} \operatorname{Im} G_{d}(\omega)=\frac{z \Gamma_{K} / \pi}{\left(\omega-\epsilon_{K}\right)^{2}+\Gamma_{K}^{2}} .
$$

In the case of the single-level AIM, the Kondo temperature $T_{K}$ can be easily calculated from the parameters of the model as follows: 6

$$
\Gamma_{K}=k T_{K}=\frac{\sqrt{\Gamma_{d} U}}{2} e^{\pi \epsilon_{d}\left(\epsilon_{d}+U\right) / \Gamma_{d} U},
$$

where $\Gamma_{d}$ is the broadening of the $d$ level due to the coupling to the bath obtained by integrating out the bath degrees of freedom,

$$
\Gamma_{d}=V^{2} \rho_{\text {bath }}(\omega=0) .
$$

Here we have assumed an approximately constant bath density of states $\rho_{\text {bath }}$ and the coupling $V$ independent of $q$. Note the exponential dependence of the Kondo temperature on the interaction $U$ and broadening $\Gamma_{d}$. This means that mild changes in the parameters can have a huge effect on the Kondo temperature. Also note that other definitions of the Kondo temperature may differ by a constant prefactor.

Since the 1 AIM is a Fermi liquid at zero temperature, ${ }^{61}$ we can exploit further relations of the Fermi liquid theory. For example, one obtains the following important relationship between the impurity-level occupation and the Kondo parameters (see, e.g., Chap. 5 in Ref. 6):

$$
n_{d}=1-\frac{2}{\pi} \arctan \left(\frac{\epsilon_{K}}{k T_{K}}\right) .
$$

Also from the Fermi liquid theory of the Anderson model we obtain the following exact relation between the density of states at the Fermi level $\epsilon_{F} \equiv 0$ (in general not the maximum of the Kondo peak) to the occupation of the $d$ level and the broadening $\Gamma_{d}$ due to the coupling of the $d$ level to the rest of the system:

$$
\rho_{d}(0)=\frac{\sin ^{2}\left(\frac{\pi}{2} n_{d}\right)}{\pi \Gamma_{d}} .
$$

Finally the amplitude of the Kondo resonance is

$$
A_{K} \equiv \rho_{d}\left(\epsilon_{K}\right)=\frac{z}{\pi k T_{K}} .
$$

\section{Underscreened Kondo effect in the multilevel Anderson model}

$\mathrm{Co}$ and $\mathrm{Fe}$ feature two and three holes, respectively, in the $3 d$ shell of each atom and, hence, have an atomic spin $S>1 / 2$ due to Hund's rule coupling. Therefore, a description in terms of a 1AIM as before is problematic. Nevertheless, the experimental results can be fitted very well to a 1AIM (see below and Sec. V). The explanation might be that we are really dealing with a so-called underscreened Kondo effect ${ }^{62}$ (UKE) where only a spin-1/2 in one of the $d$ levels is screened while the rest remains unscreened.

Such a UKE behaves in many ways like a normal (fully screened) $S=1 / 2$ Kondo effect. For example, it is still characterized by a zero-bias anomaly resulting from a sharp resonance in the screened impurity level. Further support for this hypothesis comes from a paper by Perkins et al.: ${ }^{63}$ They find that for an underscreened Kondo lattice, ferromagnetism and the Kondo effect can, in fact, coexist. On the other hand, the UKE has a number of peculiar consequences, such as the formation of a so-called singular Fermi liquid state characterized, e.g., by the divergence of the quasiparticle weight and thermodynamic quantities, such as the specific heat capacity. ${ }^{64,65}$ Note that, in this sense, the UKE is very similar to the so-called ferromagnetic Kondo effect, ${ }^{66}$ where the impurity spin couples ferromagnetically with the conduction electrons. The resulting antiscreening of the magnetic moment by the conduction electrons also leads to the formation of a singular Fermi liquid state. The ferromagnetic Kondo effect has recently been discussed theoretically in the context of magnetic impurities in nanocontacts. ${ }^{67,68}$

We consider a two-level Anderson impurity model (2AIM). This should model the situation of Co which has two holes in the $d$ shell. As in the case of the simple AIM, the system is divided into two subsystems: the conduction electron bath $\mathrm{B}$ and the impurity I with the two interacting levels. The version of the 2AIM model that is relevant for the underscreened Kondo effect is depicted schematically in Fig. 3(a): Only one of the impurity levels couples to the conduction electron bath while the coupling of the other level is negligible. ${ }^{69,70}$ Hence, 
(a)
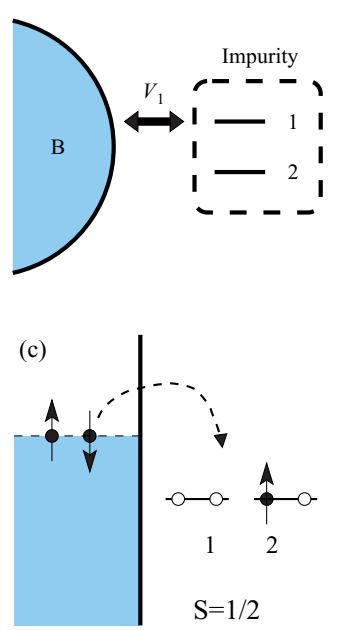
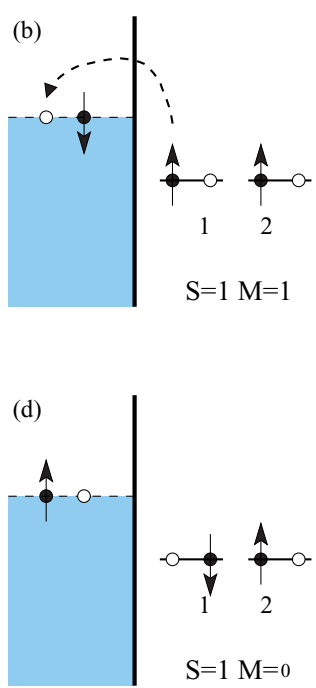

FIG. 3. (Color online) (a) Schematic drawing of the two-level Anderson impurity model in the underscreened situation. Only level 1 of the impurity is coupled to the bath B. [(b)-(d)] Schematic illustration of a hopping process contributing to the underscreened Kondo effect in the two-level Anderson impurity model.

the Hamiltonian of the 2AIM is given by

$$
\begin{aligned}
\hat{H}_{2 \mathrm{AIM}}= & \hat{H}_{I}+\hat{H}_{B}+\hat{H}_{T}=\sum_{i}\left(\epsilon_{i} \hat{n}_{i}+U \hat{n}_{i \uparrow} \hat{n}_{i \downarrow}\right) \\
& +U^{\prime} \hat{n}_{1} \hat{n}_{2}-J_{H} \hat{\mathbf{s}}_{1} \cdot \hat{\mathbf{s}}_{2}+\sum_{q, \sigma} \epsilon_{q} c_{q \sigma}^{\dagger} c_{q \sigma} \\
& +\sum_{q, \sigma} V_{1, q}\left(d_{1 \sigma}^{\dagger} c_{q \sigma}+c_{q \sigma}^{\dagger} d_{1 \sigma}\right),
\end{aligned}
$$

where $d_{i \sigma}\left(d_{i \sigma}^{\dagger}\right)$ destroys (creates) one electron in impurity level $i$ with spin $\sigma$, and $\hat{n}_{i \sigma}=d_{i \sigma}^{\dagger} d_{i \sigma}$ is the occupation number operator for level $i$ and spin $\sigma$ and $\hat{n}_{i}=\hat{n}_{i \uparrow}+\hat{n}_{i \downarrow}$. $\hat{\mathbf{s}}_{i}=\sum_{\sigma \sigma^{\prime}} d_{i \sigma}^{\dagger} \vec{\tau}_{\sigma \sigma^{\prime}} d_{i \sigma^{\prime}}$ measures the spin in level $i . \epsilon_{i}$ are the energies of the two impurity levels, $U$ is the Coulomb repulsion within the same level $i$, and $U^{\prime}$ is the Coulomb repulsion between electrons in different levels which is generally smaller than $U$, and $J_{H}$ is the Hund's rule coupling. The bath B is described as in the case of the 1AIM, Eq. (1). Finally, only impurity level 1 is coupled to the conduction electron bath with hopping $V_{1}$, while the coupling of impurity level 2 is negligible.

For the sake of simplicity, we also assume that the two impurity levels are degenerate: $\epsilon_{1}=\epsilon_{2}=\epsilon$. Typically, the intralevel Coulomb repulsion $U$ is bigger than the interlevel Coulomb repulsion $U^{\prime}$ by an amount of the order of the Hund's rule coupling: $U \approx U^{\prime}+J_{H}$. Assuming a constant bath density of states and $q$-independent coupling $V_{1, q}=V_{1}$, the half-width of level 1 due to the coupling to the conduction electrons is given by $\Gamma_{1}=V_{1}^{2} \rho_{\text {bath }}$.

In the situation where the two impurity levels are well below the Fermi energy of the conduction electrons $\left(\epsilon_{F}>2 \epsilon+U^{\prime}\right)$, and the intralevel Coulomb repulsion $U$ is strong enough to prevent double occupation of each impurity level $(2 \epsilon+U>$ $\left.\epsilon_{F}\right)$, the impurity will be doubly occupied. And, due to Hund's rule coupling, the impurity will then be in a total spin-triplet

state, i.e., will have total spin $S_{\mathrm{I}}=1$ (see Appendix A for further details).

In this situation, switching on the coupling $H_{T}$ between the impurity and the conduction electron bath gives rise to hopping processes as depicted schematically in Fig. 3 which will partially screen the total spin 1 of the impurity by flipping the spin in impurity level 1 . This partial screening of the impurity spin $S>1 / 2$ by a single conduction electron channel is called the UKE. ${ }^{62}$ The coupling to the residual spin in the other impurity level gives rise to a so-called singular Fermi liquid (SFL) behavior, ${ }^{64,71,72}$ in contrast to the normal Fermi liquid behavior of the usual fully screened Kondo effect.

The SFL is characterized by a cusp in the spectral density at low temperatures, i.e., for low energies the spectral density of impurity level 1 is approximately given by ${ }^{70,73}$

$$
\rho_{1}(\omega) \approx \frac{1}{\pi \Gamma_{1}}\left(1-\frac{b}{\ln \left(|\omega| / k T_{0}\right)^{2}}\right)
$$

where $T_{0}$ is a new temperature scale associated with the spin- 1 $\mathrm{UKE}$ and $b>0$ is a constant. The cusp in the spectral density is related to a logarithmic divergence of the quasiparticle weight in the underscreened Kondo regime: ${ }^{65} z \propto 1 / \omega \log \left(k T_{0} / \omega\right)$. Hence, in contrast to the normal Kondo effect, there is no well-defined quasiparticle associated with the UKE, hence, the name singular Fermi liquid. Note that, although the quasiparticle weight $z$ diverges for $\omega \rightarrow 0$, the spectral density $\rho_{1}(\omega)$ itself does not diverge.

In an actual experiment the logarithmic cusp characteristic for the UKE is probably hard to resolve due to limited resolution and the smoothening effect of finite temperature. Hence, in practice, the zero-bias anomaly arising from the UKE is undistiguishable from that arising from the normal Lorentzian-type Kondo peak unless very low temperatures can be reached and the experimental resolution is fine enough to resolve the cusp.

The half width of the resulting UKE resonance is determined by the temperature $T_{0}$ as $\Gamma_{K}^{S=1}=k T_{0} \exp (-\sqrt{2 b})$. In order to compare this width with the one of the normal Kondo peak in the 1AIM, we consider the particle-hole symmetric regime. Following Ref. 70 we have $k T_{0} \propto \exp \left[-\frac{\pi}{4 \Gamma_{1}}(U+\right.$ $\left.\left.J_{\mathrm{H}} / 2\right)\right]$ while $k T_{K} \propto \exp \left[-(\pi U) /\left(4 \Gamma_{d}\right)\right]$. Hence, for the same parameters $U$ and $\Gamma_{d}=\Gamma_{1}$, the width of the resonance should be smaller in the case of the UKE than for the normal Kondo effect due to Hund's rule coupling $J_{H}$ and the additional exponential factor $\exp (-\sqrt{2 b})<1$. Assuming that the interaction $U$ and coupling $\Gamma$ for the $d$ level giving rise to the Kondo effect is approximately the same for the three transition metals considered here, this might explain why the widths of the zero-bias anomalies obtained for the cases of $\mathrm{Fe}$ and $\mathrm{Co}$ are considerably smaller than for $\mathrm{Ni}$ (see Sec. V).

Finally, for the 2AIM in the UKE regime one obtains exactly the same formula relating the occupation $n_{1}$ of the impurity level 1 (the one coupled to the conduction electron bath) to the width and position of the resonance as in the case of the 1AIM, Eq. (6). ${ }^{70}$ This explains why the results in the cases of $\mathrm{Co}$ and Fe can be fitted so well to the formula for the 1AIM (see Sec. V). 


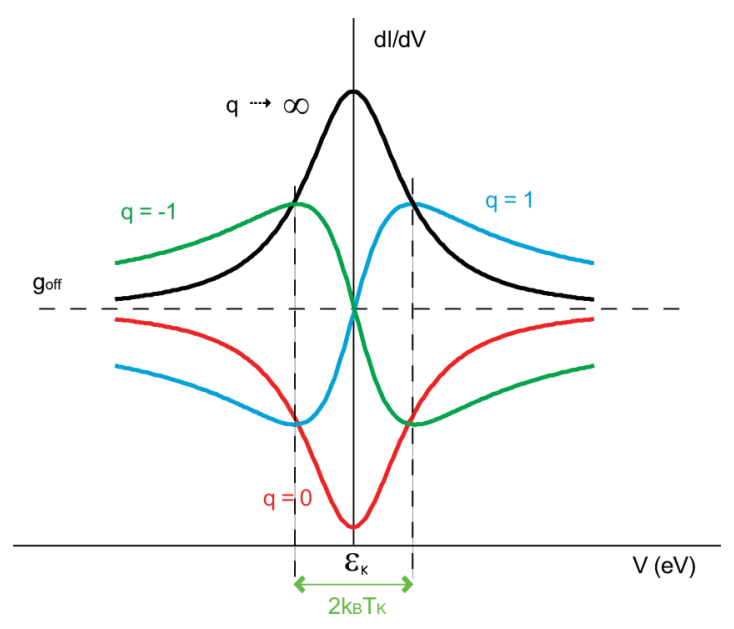

FIG. 4. (Color online) Graphical definition of the Fano resonance parameters.

\section{Kondo-Fano line shapes}

The goal of this section is to devise a simple model in order to understand the occurrence of different Fano line shapes (i.e., peaks, dips, or asymmetric Fano curves) for the same material. Our simple model also demonstrates the complicated dependence of the Fano parameters on the basic microscopic parameters. Fano line shapes in a related model have recently been studied by Zitko $^{74}$ using the numerical renormalization group and focusing on the temperature dependence of the Fano line shapes. Here we neglect any temperature effects since the measured Kondo scales are much higher than the temperature of $4.2 \mathrm{~K}$, at which the experiments were performed. In any case, the goal of this section is not to give an exact description of the conductance spectra but rather to obtain a qualitative understanding of how different line shapes emerge and how they depend on the microscopic details of the system.

In the following we will show that the low-bias conductance $\left(\|e V\| \leqslant \Gamma_{K}\right.$ ) of our simplified model of a nanocontact shown in Fig. 2 is well described by the Fano formula,

$$
G(V)=g_{\text {off }}+\frac{A}{1+q^{2}} \frac{(\epsilon+q)^{2}}{\epsilon^{2}+1} \text { with } \epsilon=\frac{e V-\epsilon_{K}}{\Gamma_{K}},
$$

where $g_{\text {off }}$ is the conductance offset, $A$ is the amplitude of the Fano resonance, $q$ is the Fano factor determining the shape of the Fano resonance (see Fig. 4), and, as described before, $\epsilon_{K}$ is the energy position and $\Gamma_{K}$ the half-width of the Kondo peak in the $d$ level which, thus, determine the position and width of the resulting Fano line shape.

As can be seen from Fig. 4, when the Fano factor becomes very large $(q \rightarrow \infty)$, the conductance has a Lorentzian line shape. This is the case when the coupling $V_{s d}$ between the $s$ and the $d$ levels of our tip atom is negligible so the zero-bias anomaly in the conductance is dominated by the direct transmission through the Kondo resonance of the $d$ level. On the other hand, for $q=0$ we obtain a dip feature in the conductance. This is, for example, the case when the direct transmission through the $d$ channel becomes negligible (e.g., for $\Gamma_{R, d} \approx 0$ ) so the conductance is only given by the $s$ channel, which features a Lorentzian dip due to the coupling $V_{s d}$ to the Kondo resonance in the $d$ level. For $|q|=1$ the Fano formula gives the typical asymmetric line shapes. In this case, the conductance is also dominated by the $s$ channel coupled to the Kondo resonance in the $d$ level as for $q=0$ but now the $s$ level of the tip atom is not near the Fermi level.

In the Appendix B we give a derivation of the Fano formula (11) for our simplified model of a nanocontact shown in Fig. 2: The (left) tip atom where the Kondo effect is taking place is modeled by one $s$ level and one $d$ level. While the $s$ level couples well to both electrodes (via $\Gamma_{L, s}$ and $\Gamma_{R, s}$ ) and, thus, has a nearly perfect transmission, the $d$ level hosting the Kondo resonance has a much weaker coupling to both electrodes $\left(\Gamma_{L, d}\right.$ and $\left.\Gamma_{R, d}\right)$. Generally, we assume that the couplings to the left electrode are stronger than to the right electrode, i.e., $\Gamma_{L, \alpha}>$ $\Gamma_{R, \alpha}$. Additionally, there is a small hybridization $V_{s d}$ between the $s$ and the $d$ levels due to the crystal field.

As Meir and Wingreen showed in their landmark paper, at zero temperature and in linear response the conductance through a nanoscopic conductor is well described by the Landauer formula, even in the case of a strongly interacting system. ${ }^{75}$ The conductance $G$ for small bias $V$ is then given in terms of the quantum mechanical transmission function $T(\omega)$ as $G(V)=G_{0} \times T(e V)$, where $G_{0}=2 e^{2} / h$ is the fundamental conductance quantum.

As shown in the Appendix B, the transmission $T(\omega)$ through the tip atom can be decomposed into the contributions of direct transmission through the indivdiual $s$ and $d$ channels, namely $T_{s}(\omega)$ and $T_{d}(\omega)$, respectively, and a mixed channel involving hopping between both channels, $T_{s d}(\omega)$,

$$
T(\omega)=T_{s}(\omega)+T_{d}(\omega)+T_{s d}(\omega) .
$$

Following Eqs. (B8) and (B9), the direct channel transmissions $T_{s}$ and $T_{d}$ are given by the spectral densities of the $s$ and $d$ levels, respectively, and the couplings of the $s$ and $d$ levels to both electrodes $L$ and $R$.

The Kondo effect gives rise to the appearance of a Kondo resonance in the the $d$-level spectral function $\rho_{d}(\omega)$ given by Eq. (3). Hence, the contribution of the $d$ channel to the total transmission has a Lorentzian line shape,

$$
T_{d}(\omega)=\frac{z^{2} \Gamma_{L, d} \Gamma_{R, d}}{\left(\omega-\epsilon_{K}\right)^{2}+\Gamma_{K}^{2}}=\frac{4 \Gamma_{L, d} \Gamma_{R, d}}{\Gamma_{L, d}+\Gamma_{R, d}} \frac{1}{1+x^{2}},
$$

where we have used $\Gamma_{K}=z\left(\Gamma_{L, d}+\Gamma_{R, d}\right) / 2$ and we have defined the dimensionless quantity $x=\left(\omega-\epsilon_{K}\right) / \Gamma_{K}$. Hence, as pointed out above, the $d$-channel contribution to the transmission can only give rise to Fano line shapes with $q \rightarrow \infty$.

We assume that the unperturbed $s$ channel (i.e., without coupling to the $d$ level) has a featureless (i.e., flat) and almost perfect transmission $T_{s}^{0} \approx 1$. Due to the coupling $V_{s d}$ to the $d$ level, this transmission is modified according to (see Appendix B),

$$
T_{s}(\omega)=T_{s}^{0}\left[1+\frac{2 z V_{s d}^{2}}{\Gamma_{s} \Gamma_{K}} \frac{\left(x+q_{0}\right)^{2}-\left(x^{2}+1\right)}{\left(x^{2}+1\right)\left(1+q_{0}^{2}\right)}\right],
$$

where $\Gamma_{s}=\Gamma_{L, s}+\Gamma_{R, s}$ is the total broadening of the $s$ level and the dimensionless quantity $q_{0}$ has been defined as the ratio between the $s$-level energy and the $s$-level broadening: $q_{0}=$ $-2 \epsilon_{s} / \Gamma_{s}$ (see Appendix B for details). The second term of the right-hand side is of the Fano form, Eq. (11), and represents 
the modulation of the almost perfectly transmitting $s$ channel due to the coupling to the Kondo resonance in the $d$ channel. Since generally $2\left|\epsilon_{s}\right|<\Gamma_{s}$ we should have $\left|q_{0}\right| \leqslant 1$ and, therefore, the $s$-channel contribution to the transmission (14) can only give rise to diplike $\left(q_{0} \approx 0\right)$ or asymmetric Fano line shapes $q \approx 1$ but not to the peak line shapes where $\left|q_{0}\right| \gg 1$.

For the mixed-channel contribution $T_{s d}$ to the total transmission we find the following expression in the Appendix B:

$$
T_{s d}(\omega)=T_{s}^{0} \frac{z^{2} V_{s d}^{2}}{\Gamma_{K}^{2}}\left(\frac{\Gamma_{R, d}}{\Gamma_{R, s}}+\frac{\Gamma_{L, d}}{\Gamma_{L, s}}\right) \frac{1}{1+x^{2}} .
$$

This contribution describes transmission processes where an electron hops from one electrode to the $s$ level of the tip atom, subsequently to the $d$ level via $V_{s d}$, and then to the other electrode. Due to the Kondo peak in the $d$ level, it gives rise to a Lorentzian line shape in the transmission.

Hence, we have shown that our model can give rise to all possible Fano line shapes as obtained in the experiments. More specifically, the $s$-channel contribution $T_{s}(\omega)$ can give rise to the diplike features $(q \approx 0)$ and the asymmetric Fano features $(|q| \approx 1)$ while the $d$-channel contribution $T_{d}(\omega)$ and the mixed-channel contribution $T_{s d}(\omega)$ give rise to Lorentzian line shapes $(|q| \rightarrow \infty)$ in the transmission. Which term dominates depends on the specific amplitudes of the different transmission channels given by the basic parameters of our model.

It is, of course, possible to achieve the "canonical" Fano line-shape form for the conductance as in Eq. (11) by summing up all the individual contributions to the total transmission (12) and reorganizing the terms. We then obtain a "new" Fano factor $q$ that differs from the Fano factor $q_{0}$ for the pure $s$ channel contribution (14). This new Fano factor will depend on $q_{0}$ and the amplitudes of the individual contributions to the transmission and yields a relatively complicated expression in terms of the basic parameters of our model. The same is true for the amplitude $A$ of the Fano feature defined by Eq. (11).

However, we can obtain quite simple expressions for $q$ and $A$ in Eq. (11) in an important limit of our model, namely when the coupling of the $d$ level giving rise to the Kondo peak to one of the electrodes becomes very small, e.g., $\Gamma_{R, d} \rightarrow 0$. In that case, the direct transmission through the $d$ channel is strongly suppressed, i.e., $T_{d} \approx 0$, so now only the mixedchannel contribution $T_{s d}$ can give rise to a Lorentzian line shape in the transmission,

$$
\begin{gathered}
q=\sqrt{\frac{\Gamma_{s}}{\Gamma_{L, s}}} q_{0}=-\frac{2 \epsilon_{s}}{\sqrt{\Gamma_{s} \cdot \Gamma_{L, s}}}, \\
A=\frac{1+\Gamma_{s} / \Gamma_{L, s}}{1+q_{0}^{2}} \frac{4 V_{s d}^{2}}{\Gamma_{s} \Gamma_{L, d}}=2 \pi \frac{\Gamma_{s}}{\Gamma_{L, s}} \frac{\Gamma_{L, s}+\Gamma_{s}}{\Gamma_{s}^{2}+4 \epsilon_{s}^{2}} V_{s d}^{2} A_{K},
\end{gathered}
$$

where $A_{K}$ is the amplitude of the Kondo resonance in the spectral function of the $d$ level as given by Eq. (8). Note that the Fano factor $q_{0}$ of the $s$-channel transmission $T_{s}$ is now scaled by the factor $\sqrt{\Gamma_{s} / \Gamma_{L, s}}>1$ to yield the Fano factor $q$ of the Fano line shape in Eq. (11), meaning that, for large values of the ratio $\sqrt{\Gamma_{s} / \Gamma_{L, s}}$, we can obtain $|q|$ values $>1$ for $0<\left|q_{0}\right| \leqslant 1$. Furthermore, we see that the amplitude of the Fano resonance in the conductance is proportional to the amplitude of the Kondo resonance and to the square of the coupling $V_{s d}$ between the $s$ level and the $d$ level of the tip atom.

\section{EXPERIMENTAL DETAILS}

The experiments were performed using a home-made STM operated in a He cryostat at $4.2 \mathrm{~K}$. Two pieces of the same metal wire $(\mathrm{Fe}, \mathrm{Co}$, or $\mathrm{Ni})$ of $0.1 \mathrm{~mm}$ of diameter were scratched and sonicated in acetone and isopropanol before being mounted as "tip and sample" in the microscope. The conductance between the two pieces of metal is obtained in a two-terminal configuration by measuring the current at a fixed bias voltage, in this case, $100 \mathrm{mV}$. In these conditions, we can record traces of conductance wile changing the distance between the two metals [a typical trace is shown in Fig. 1(a)] in a similar fashion as performed in other break-junction experiments. ${ }^{40}$ The samples are then prepared at low temperatures by indentation until no traces with subquantum events are shown. The histograms [Fig. 1(b)] are similar to those at mechanically controlled break-junction (MCBJ) experiments where a fresh surface is formed at cryogenic vacuum when breaking a notched wire by the controlled bending of a elastic substrate (see, e.g., Ref. 40 for details). This shows that our measurements are performed over a clean spot of our samples. A strong indentation between the two electrodes is performed between the fabrication of consecutive atomic contacts to ensure the cleanliness. Our STM setup has imaging capabilities; however, our surfaces are not atomically flat due to preparation and in order to acquire a large number of contacts to analyze and no imaging is performed between contact formation.

As described in Sec II, traces show plateaus coming from the atomic rearrangement of the wire while pulling and the last plateau around the quantum of conductance is associated with the formation of a single atom contact. Once the conductance of the one-atom contact has been determined by the position of the first peak of the conductance histogram, we can fabricate monoatomic contacts by stopping the breaking process of the contact at the desired value of conductance within the range defined by the conductance histogram. The stability of our system allows us to maintain such an atomic contact for hours. We study the transport spectroscopy of these contacts in a similar way as performed in other transport experiments either in tunneling or high-conductance regimes (e.g., the case of quantum dot devices or the spectroscopy of adatoms by STM). We sweep the bias voltage from -100 to $100 \mathrm{mV}$ while recording the $d I / d V$ signal with the help of a lock-in amplifier when adding a $1-\mathrm{mV}$ ac excitation at a frequency about $1 \mathrm{kHz}$ to the applied bias voltage.

\section{RESULTS}

The fabrication of atomic contacts by using a STM (or MCBJ) offers the possibility of studying a high number of different contact configurations in a reasonable amount of time. We fabricated hundreds of atomic contacts of $\mathrm{Fe}, \mathrm{Co}$, and $\mathrm{Ni}$ and performed electron spectroscopy measurements as described in Sec. IV. About $80 \%$ of these curves showed clear asymmetric profiles centred at zero bias as the ones plotted in Fig. 5. 

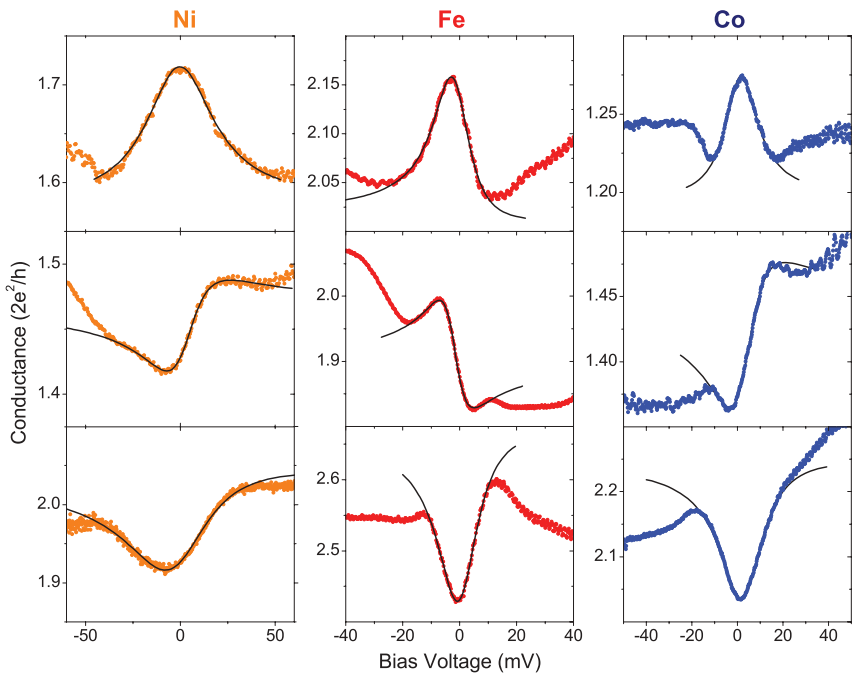

FIG. 5. (Color online) Representative differential conductance curves for atomic contacts of $\mathrm{Fe}, \mathrm{Co}$, and $\mathrm{Ni}$. A characteristic resonance associated with the Kondo effect appears at small bias. The corresponding fit to the Fano line shape is shown in black for each of these resonances. Due to the large differences in the width of the resonances, the fitting range is chosen so it is possible to fit the whole range of the resonance to the Fano line shape. The selected curves exemplify different symmetry cases, from the more symmetric ( $q \simeq \infty$ and $q \simeq 0$, respectively) to the clearly asymmetric ones $(q \simeq \pm 1)$. While all possible symmetries seem to happen for the three materials, the width of the resonance which is associated with the Kondo temperature differs for the three materials, especially for $\mathrm{Ni}$.

Similar asymmetric zero-bias anomalies (ZBA) were reported for ferromagnetic atomic contacts and attributed to the existence of conductance fluctuations ${ }^{76}$ or to the existence of a magnetic domain wall. ${ }^{77}$ On the other hand, these asymmetric profiles resemble the data reported for single magnetic adatoms in the contact regime, not only in shape but also in the energy scale of the features. ${ }^{29,30}$

In our previous work we have shown clear evidence of the Kondo effect being responsible for these ZBA. ${ }^{38}$ Recently, ZBA profiles, possibly of similar origin, have been reported in Refs. 78 and 79 for nanocontacts made from other materials and also show the Kondo-related ZBA in adatoms contacted by ferromagnetic tips. ${ }^{36,37}$

As in other Kondo systems, the asymmetric line shapes can be fitted to the Fano equation (11). From this fitting, we extract the values for the different parameters that describe the Kondo effect. As sketched in Fig. 4, the width of the resonance is directly related to the Kondo temperature $\left(T_{K}\right)$. The Fano parameter $q$ contains information about the symmetry of the line shape. We denote by $\epsilon_{K}$ the energy at which the resonance is centered. As introduced in Sec. III, this parameter is associated with the energy position of the Kondo resonance and, therefore, to the occupation of impurity level $n_{d}$, Eq. (6). Finally, $A$ is the amplitude of the Fano profile and $g_{\text {off }}$ the conductance offset of the curve from the resonance.

Each realization of the contact leads to a slightly different configuration. The statistical distributions of the different parameters of the Fano equation described above will reflect the subtle differences in the electronic structure of each contact. We present below a statistical analysis of each of these parameters for hundreds of contacts of $\mathrm{Co}, \mathrm{Fe}$, and $\mathrm{Ni}$. This novel statistical analysis (since in our previous work ${ }^{38}$ we analyzed only briefly the shape and mean value of the Kondo temperature) together with theoretical considerations brings new insight into the physics of Fano-Kondo resonances. We present now the distributions of these parameters and compare the results among materials.

\section{A. Kondo temperatures}

As described in Sec. III, the zero-bias resonances observed in the conductance characteristics of Kondo systems are directly related to the resonances developed in the spectral density of the system. As explained in Sec. III, the width of this resonance is determined by the energy scale of the Kondo screening, the so-called Kondo temperature $T_{K}$. Thus, the width of the observed Fano line shapes must be proportional to the Kondo temperature of the system. More precisely, we define the Kondo scale as the half width of the Fano line shape: $\Gamma_{K}=k_{B} T_{K}$. The width of the measured Kondo resonance is strongly affected by a finite temperature of the system: In addition to the standard thermal broadening of any differential conductance feature, the Kondo resonance presents an intrinsic thermal broadening. ${ }^{24}$ This results in a considerable extra broadening of the resonance at temperatures on the order of magnitude of $T_{K}$. In our case, since the width of our resonances excesses in more than an order of magnitude the experimental temperature of $4.2 \mathrm{~K}$ and the bias voltages used are low enough ${ }^{35}$ we can disregard thermal effects and consider that we can extract the Kondo temperature for each contact directly from the width of the Fano resonance.

As we have already described in Ref. 38, the distribution of Kondo temperatures fits a logarithmic normal distribution for the three materials $\mathrm{Co}, \mathrm{Fe}$, and $\mathrm{Ni}$, meaning that the logarithm of $T_{K}$ is normally distributed (presented in Fig. 6). This peculiar behavior is easily understood when interpreted in terms of the Kondo effect: Since many different atomic configurations result in single-atom contacts, their electronic properties, such as conductance (Fig. 1), density of states, and the associated energy scales, are expected to be normally distributed. On the other hand, following Eq. (4) the Kondo temperature depends exponentially on the typical energy scales of the problem. Hence, $\ln T_{K}$ for different contacts should follow a normal distribution if the relevant energy scales of the problem are normally distributed.

By just looking at the resonances, as, for example, the ones shown in Fig. 5, it can be observed that the Fano features in the conductance spectra of $\mathrm{Ni}$ contacts are considerably broader than the ones for the cases of Co and Fe. As shown in Fig. 6 and summarized in Table I, the histograms yield most frequent values for the resonance widths of $T_{K}=90 \mathrm{~K}, 120 \mathrm{~K}$, and $280 \mathrm{~K}$ for $\mathrm{Fe}, \mathrm{Co}$, and $\mathrm{Ni}$, respectively, following the same trend $T_{K}^{\mathrm{Fe}}<T_{K}^{\mathrm{Co}}<T_{K}^{\mathrm{Ni}}$ as in the case of adatoms of these elements deposited on nonmagnetic surfaces ${ }^{25}$ and diluted alloys of $\mathrm{Cu}$ containing the same concentration of magnetic atoms. ${ }^{80}$ In simple terms, the Kondo temperature decreases as the size of the screened magnetic moment increases as we go from $\mathrm{Ni}$ to $\mathrm{Fe}$. 


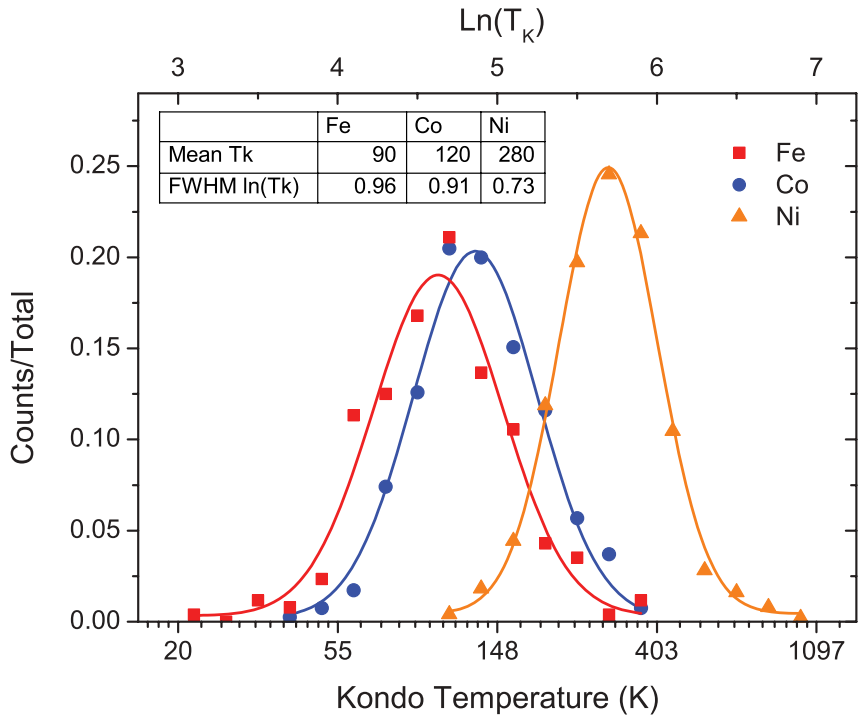

FIG. 6. (Color online) Histograms of values of logarithm of Kondo temperature for $\mathrm{Fe}, \mathrm{Co}$, and $\mathrm{Ni}$. The histograms are normalized to the total number of counts. The distribution of values for the $\ln (\mathrm{Tk})$ is clearly narrower for $\mathrm{Ni}$ than for $\mathrm{Fe}$ and $\mathrm{Co}$ in spite of the higher value of Kondo temperatures for $\mathrm{Ni}$, as summarized in the inset. The most frequent value of the Kondo temperature is determined with an error of $10 \mathrm{~K}$.

Figure 6 shows the distribution for $\ln \left(T_{K}\right)$ fitted to a Gaussian distribution. Surprisingly enough, in spite of the considerably higher values of Kondo temperatures, the distribution of Ni when plotted in logarithmic scale is considerably narrower than in the cases of $\mathrm{Fe}$ and $\mathrm{Co}$ (see also inset of Fig. 6). This suggests that, in the case of $\mathrm{Ni}$, the characteristics of the Kondo screening differ markedly from the cases of Co and $\mathrm{Fe}$, possibly indicating a different mechanism for the case of $\mathrm{Ni}$ and the cases of $\mathrm{Co}$ and $\mathrm{Fe}$.

The higher Kondo temperatures for $\mathrm{Ni}$ as well as their narrower distribution could well be connected to the different chemical valence and the resulting magnetic moment in comparison to $\mathrm{Co}$ and $\mathrm{Fe}$ : While Ni basically has one hole in the $3 d$ shell and, therefore, features an atomic spin of $1 / 2$, Co and $\mathrm{Fe}$ have two and three holes in their $3 d$ shells associated with atomic spins of 1 and $3 / 2$, respectively. Hence, in the cases of $\mathrm{Co}$ and $\mathrm{Fe}$, the possibility exists that the full atomic

TABLE I. A comparison of Kondo temperatures in the case of magnetic impurities in bulk $\mathrm{Cu}$, magnetic adatoms in the tunneling regime, and for the ferromagnetic contacts ( $\mathrm{Fe}, \mathrm{Co}$, and $\mathrm{Ni}$ ) in this work. The different values of the Kondo temperatures in the case of adatoms correspond to measurements performed over different substrates. Also shown are the magnetic moments of isolated atoms and in bulk.

\begin{tabular}{|c|c|c|c|}
\hline & $\mathrm{Fe}$ & Co & $\mathrm{Ni}$ \\
\hline$T_{K}(\mathrm{~K})$ in bulk $\mathrm{Cu}^{80}$ & $10-50$ & $300-700$ & $\simeq 1000$ \\
\hline$T_{K}(\mathrm{~K})$ adatoms & - & $53-92^{23}$ & $120^{25}$ \\
\hline$T_{K}(\mathrm{~K})$ this work & 90 & 120 & 280 \\
\hline$m_{\text {atom }}(\mu B)$ & 3 & 2 & 1 \\
\hline$m_{\text {bulk }}(\mu B)$ & 2.22 & 1.72 & 0.60 \\
\hline
\end{tabular}

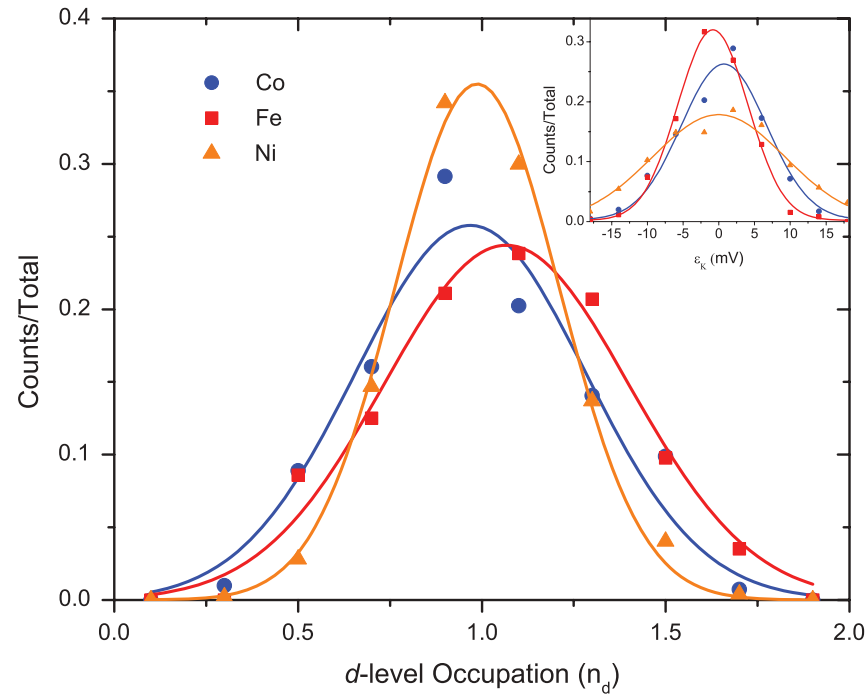

FIG. 7. (Color online) Histograms of the $d$-level occupations $n_{d}$ for contacts of $\mathrm{Fe}, \mathrm{Co}$, and $\mathrm{Ni}$. The colored lines show the fitting of these distributions to a Gaussian peak. Surprisingly, the distribution for $\mathrm{Ni}$ is narrower than that for the other two, in spite of the fact that this material shows a broader distribution of the parameter $\epsilon_{K}$. (Inset) Distribution of $\epsilon_{K}$ for hundreds of contacts of $\mathrm{Fe}, \mathrm{Co}$, and $\mathrm{Ni}$. This parameter accounts for the deviation of the center of the Fano resonance from zero bias and is related to the position of the localized magnetic moment in the Kondo model.

spin $S>1 / 2$ is only partially screened while the spin-1/2 in the case of $\mathrm{Ni}$ is fully screened by the conduction electrons. The cases of $\mathrm{Co}$ and $\mathrm{Fe}$ would then resemble the situation of an underscreened Kondo lattice where the remaining unscreened spin couples ferromagnetically to the spins on neighboring atoms, as discussed in Ref. 63. As explained in Sec. III, such an underscreened Kondo effect is characterized by sharper resonances in comparison to the normal fully screened Kondo situation and, hence, results in lower Kondo temperatures in the analysis. In this sense, the Kondo temperatures may be underestimated in the cases of Co and Fe. We discuss this in detail in the discussion Sec. VI together with the distribution of other parameters.

\section{B. Resonance energy $\left(\epsilon_{K}\right)$ and $d$-level occupation}

Another important parameter to study is the position of the Kondo resonance $\epsilon_{K}$, which accounts for the deviation of the center of the Fano resonance from zero bias and is related to the energy of the effective Kondo level with respect to the Fermi energy. ${ }^{23}$ The inset of Fig. 7 shows the distribution of this parameter for hundreds of contacts of the three materials under study. These distributions fit a Gaussian line shape which is considerably broader for the case of $\mathrm{Ni}$ than for $\mathrm{Co}$ and $\mathrm{Fe}$.

Most interestingly, from the values of $\epsilon_{K}$ and $T_{K}$ it is possible to extract the occupation of the $d$ level (giving rise to the Kondo resonance) from the experimental data, assuming a Fermi liquid approximation as explained in Sec III, Eq. (6). This approximation should be valid here since we are well below the Kondo temperature of our system. As explained in Sec. III, this relation is even valid for the underscreened Kondo effect in the multiorbital Anderson model (although, 
TABLE II. Most frequent value of resonance energy and $d$-level occupations and their respective width of their Gaussian distributions for $\mathrm{Fe}, \mathrm{Co}$, and $\mathrm{Ni}$.

\begin{tabular}{lccc}
\hline \hline & $\mathrm{Fe}$ & $\mathrm{Co}$ & $\mathrm{Ni}$ \\
\hline Mean $\epsilon_{K}(\mathrm{mV})$ & -1.0 & 0.6 & 0.3 \\
FWHM $\epsilon_{K}(\mathrm{mV})$ & 14.7 & 16.3 & 25.4 \\
Mean $n_{d}$ & 1.07 & 0.97 & 0.99 \\
FWHM $n_{d}$ & 0.79 & 0.74 & 0.52 \\
\hline \hline
\end{tabular}

strictly speaking, we then have a singular Fermi liquid) where $n_{d}$ now refers to the the $d$ level whose spin is screened by the conduction electrons and $\epsilon_{K}$ and $T_{K}$ refer to the position and width of the Kondo resonance of the underscreened Kondo effect. Figure 7 shows the distribution of positions and the resulting calculated occupations $n_{d}$ for $\mathrm{Fe}, \mathrm{Co}$, and $\mathrm{Ni}$ which again follow a normal distribution.

As summarized in Table II, the mean value for the three materials is close to 1 . Interestingly, in spite of the broader distribution of values of $\epsilon_{K}$, the occupations for Ni contacts clearly show a narrower distribution. Numerically, this can be explained by the much narrower distribution of $\ln \left(T_{K}\right)$. Physically, the reason behind the narrower distribution of Kondo temperatures and occupations in the case of $\mathrm{Ni}$ might be that charge fluctuations become stronger with an increasing number of active levels, as already discussed in the seminal work of Nozieres: ${ }^{62}$ In the case of $\mathrm{Ni}$, we are most likely dealing with a single active impurity level due to a single hole in the $3 d$ shell of $\mathrm{Ni}$. Hence, the charge in this level is quite well defined, and the occupation is very close to one electron. In the cases of $\mathrm{Co}$ and $\mathrm{Fe}$, on the other hand, we should have more than one active impurity level and, therefore, the variation in the occupation of the level giving rise to the Kondo resonance is much stronger. Relatedly, a broader distribution in the occupation of the impurity level giving rise to the Kondo resonance should also give rise to a broader distribution in the Kondo temperatures since charge fluctuations strongly alter the width of the Kondo resonance. ${ }^{6}$

\section{Amplitudes}

At first glance, the distribution of amplitudes in Fig. 8 shows no clear differences between the three materials. The most frequent value of the amplitude is approximately 0.1 $2 e^{2} / h$, i.e., about $10 \%$ of the conductance of the contact. On the other hand, we find that the square root of the amplitude indeed follows a normal distribution, as can be seen from the inset of Fig. 8, which shows the statistical distributions of the square root of the amplitude for the three materials. A possible explanation is that following expression (17) the amplitude depends quadratically on the coupling $V_{s d}$ between the $s$ and $d$ levels of the tip atom. $V_{s d}$ is expected to vary strongly when the atomic configuration of the contact changes since it is induced by disorder in the contact region and is absent for perfect crystalline order. Hence, if $V_{s d}$ is normally distributed and is the parameter determining $A$ that is most strongly affected by changing the atomic configuration of the contact, one would expect $\sqrt{A}$ to be normally distributed.

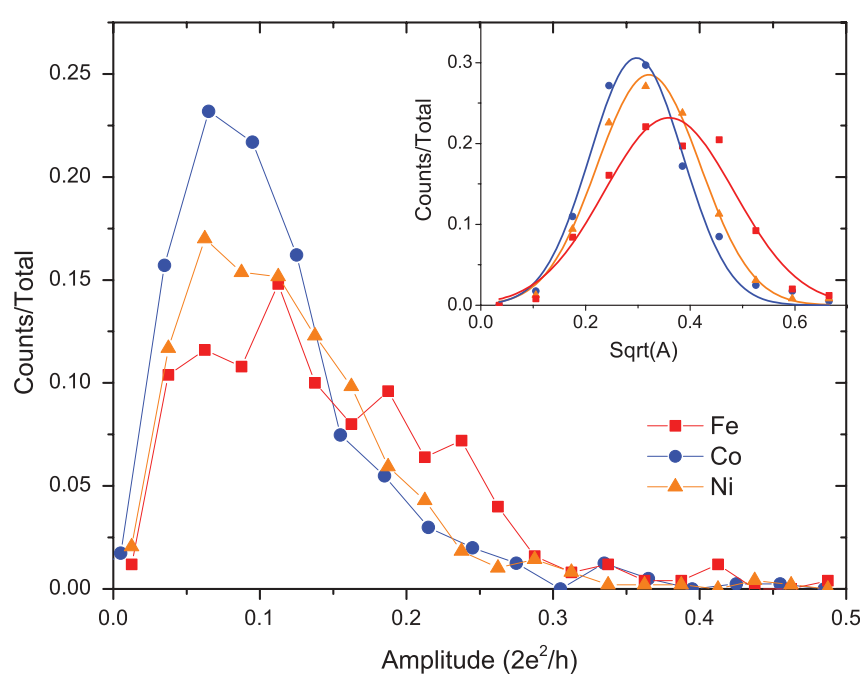

FIG. 8. (Color online) Distribution of amplitudes extracted from the fitting of characteristics of hundred of contacts of $\mathrm{Fe}, \mathrm{Co}$, and Ni to the Fano equation. The distributions are similar for the three materials, with the amplitudes being about a $10 \%$ of the conductance of the contacts. The inset shows the distribution of $\sqrt{(} A)$ and its fit to a Gaussian. This distribution could reflect the quadratic dependence of amplitude in different coupling terms.

Furthermore, one can seen that the distributions of $\sqrt{A}$ for the three materials are centered at quite similar values and also have similar widths. However, we can make out a subtle trend (Table III): The mean amplitude and the mean value of the square root of the amplitude both are slightly higher for $\mathrm{Fe}$ contacts than for the other two materials, and, moreover, both distributions are slightly broader for Fe than for $\mathrm{Co}$ and $\mathrm{Ni}$. However, this trend is not as clear as the trend observed in the distributions of Kondo temperatures $T_{K}$ for the three materials (Fig. 6). Thus, it is difficult to draw any further conclusions from it. Moreover, we would like to point out that a similar trend is observed in the distributions of the conductances (see Fig. 1 and Table III): Fe has a higher average conductance than $\mathrm{Ni}$ and $\mathrm{Co}$. More data would be needed to extract further conclusions from this analysis.

Far more interestingly, the plot of the amplitude versus the Kondo temperature presents an intriguing trend, as can be seen from Fig. 9: For all three materials there seems to be an approximately linear dependence of the amplitude on the Kondo temperature with respect to the Kondo temperature. Moreover, when normalizing the amplitudes and Kondo temperatures to the respective mean values (Table III), one obtains a universal dependence, suggesting that $A \propto f\left(T_{K}\right)$.

TABLE III. Mean values of amplitudes and width of the the Gaussian distributions of $\sqrt{A}$ and mean conductances for $\mathrm{Fe}, \mathrm{Co}$, and $\mathrm{Ni}$.

\begin{tabular}{llll}
\hline \hline & $\mathrm{Fe}$ & $\mathrm{Co}$ & $\mathrm{Ni}$ \\
\hline Mean $A$ from $\sqrt{A}$ & 0.13 & 0.088 & 0.10 \\
Mean $\sqrt{A}$ & 0.36 & 0.30 & 0.32 \\
FWHM $\sqrt{A}$ & 0.31 & 0.24 & 0.27 \\
Mean $G$ from Fig. 1 & 2.0 & 1.2 & 1.6 \\
\hline \hline
\end{tabular}



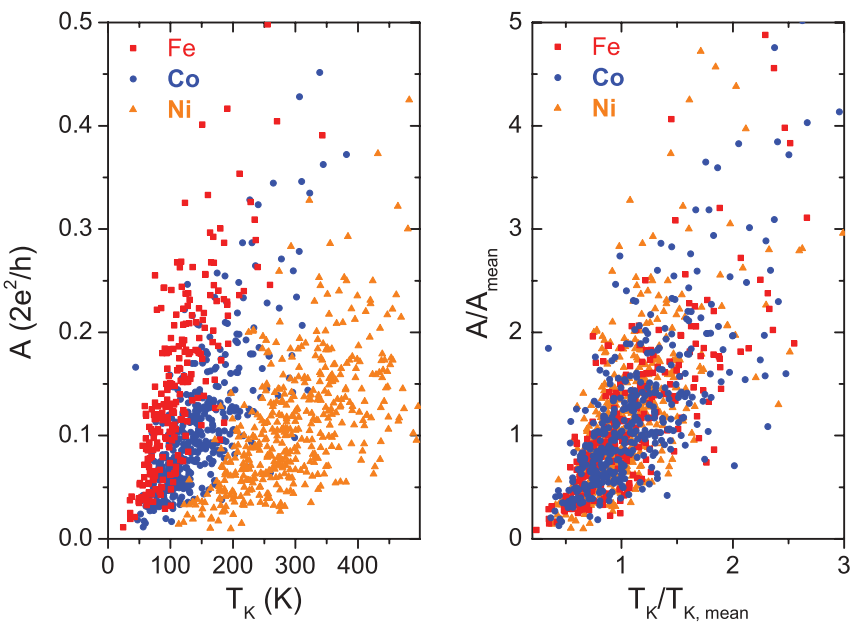

FIG. 9. (Color online) (Left) Scattered plot of the amplitude of the resonance versus the Kondo temperature obtained for hundreds of contacts of $\mathrm{Fe}, \mathrm{Co}$, and $\mathrm{Ni}$. The plot shows a clearly similar (linear) trend for the three materials. When divided by the most probable value of the Kondo temperature for each material, the distributions overlap each other, as shown in the right panel.

This universal behavior seems to reflect some kind of universality in the relation between the basic parameters $U, \epsilon_{d}$, and $\Gamma_{d}$ that ultimately determine the Kondo properties of our system. Said in another way, the basic parameters $U, \epsilon_{d}$, and $\Gamma_{d}$ are not independent of each other but are linked together in such a way that universal scaling between the amplitude $A_{K}$ and the Kondo temperature $T_{K}$ results. For example, it is conceivable that both $\Gamma_{d}$ (the coupling of the impurity level to the conduction electrons) and $U$ (the effective Coulomb repulsion of the impurity level) are related since a change in the coupling $\Gamma_{d}$ implies a change in the localization of electrons in the impurity level and, hence, can result in an alteration of the screening of the effective Coulomb interaction $U$. Further theoretical work is necessary in order to achieve a rigorous interpretation of these results.

\section{Fano parameters}

The Fano parameter $q$ accounts for the symmetry of the Fano resonances, recovering the perfect Lorentzian shape for $q \rightarrow \infty$ and its inverse for $q=0$. As already noted above, all possible symmetries are found for each material. A histogram of the values of $q$ for the three materials is shown in Fig. 10. Since $q$ ranges from 0 to $\infty$, an alternative representation where $q=\tan (\alpha)$ is chosen for simplicity. In this representation, $\alpha=0$ corresponds to the dip and $\alpha=\pi$ to the peak line shapes. The totally asymmetric cases are represented by $\alpha=\pi / 2(q=1)$ and $\alpha=3 \pi / 2(q=-1)$. There is a remarkable preference towards the symmetric cases over the more asymmetric ones in the cases of $\mathrm{Fe}$ and $\mathrm{Co}$, and, in particular, the diplike $(q=0)$ line shapes seem to prevail. For Ni, the occurrence of asymmetric cases $(|q| \sim 1)$ is considerably higher, and, overall, the Fano factors seem to be more evenly distributed than in the cases of $\mathrm{Fe}$ and $\mathrm{Co}$.

In the case of adatoms in tunneling, the symmetry is well understood in terms of the ratio between the probability of transmission through the $d$ or the $s$ channels. ${ }^{20}$ Nevertheless,

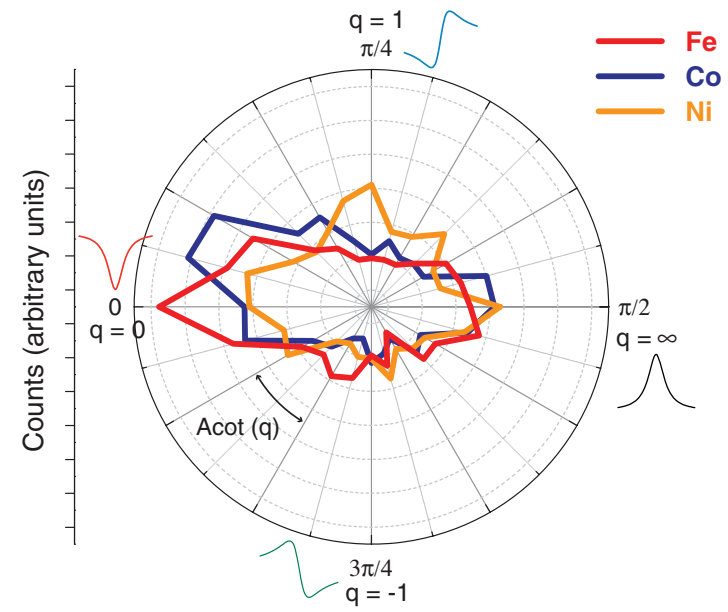

FIG. 10. (Color online) The so-called Fano parameter $q$ ranges from 0 to $\infty$, but it can be expressed as $q=\tan (\alpha)$ with $\alpha$ between 0 and $\pi$, for simplicity in the representation. $\alpha=0$ corresponds to the deep, $\alpha=\pi$ to the peak, and $\alpha=1,-1$ the totally asymmetric cases. The histograms are normalized to the total number of data.

in the point contact regime and in our case, the system presents a higher complexity. There is a totally open $s$ channel and the nature of the orbitals and couplings contributing to the interference is not completely clear. The distribution of the $q$ parameters should contain information about the contributions from the different channels to the interference which might be extracted with the help of an appropriate theoretical treatment.

In Fig. 11 we plot the evolution of a single Co contact when stretching the junction. The symmetry of the curve changes as the contact rearranges in a different manner, which translates into a different conductance of the curve (the curves are not offset). When stretching the contact of the hoppings among different orbitals, the electronic structure and, hence, the conductance varies, and this affects the

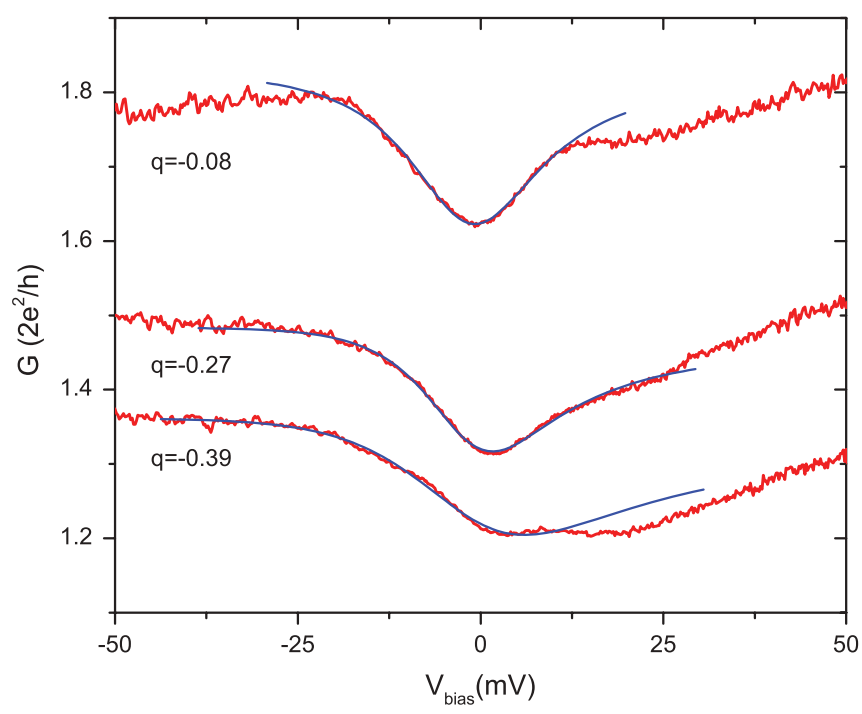

FIG. 11. (Color online) Evolution of the $d I / d V$ characteristics of a single Co contact as stretching the junction. As the conductance of the contact decreases, the symmetry of the curve, that is, the Fano parameter, $q$, also changes as a consequence of the different electronic structures of the different arrangements. 
interference between the conduction channels and also the Kondo resonance responsible for the Fano line shapes. This evolution is probably specific for each contact since an attempt to find a statistical relation between $q$ and the conductance offset results in a random distribution of points. More work is needed to fully clarify this effect.

\section{E. Summary}

In summary, the statistical analysis of the Fano-Kondo line shapes extracted from hundreds of atomic contacts shows the following.

(i) The statistical distribution of Kondo temperatures for each material is a log-normal distribution, i.e., the logarithm of the Kondo temperatures is normally distributed. This is expected in the context of the Kondo effect since the Kondo temperature depends exponentially on the different electronic properties and couplings of the system. These properties are likely to be normally distributed for the slightly different atomic configurations of the contacts. Hence, the log-normal distribution of Kondo temperature presents further evidence that the Kondo effect is responsible for the zero-bias anomalies in the conductance.

(ii) The mean value for the Kondo temperatures is considerably higher for $\mathrm{Ni}$ than for $\mathrm{Co}$ and $\mathrm{Fe}$, whereas the distribution of the logarithm of the Kondo temperature for $\mathrm{Ni}$ is narrower than for $\mathrm{Co}$ or Fe. This finding suggests that possibly a different mechanism is responsible for the Kondo screening in the case of $\mathrm{Ni}$ than in the cases of $\mathrm{Co}$ and $\mathrm{Fe}$.

(iii) The distribution of the resonance energies $\epsilon_{K}$ is Gaussian and is centered around zero for all three materials. In the case of Ni this distribution is much broader than in the cases of $\mathrm{Fe}$ and $\mathrm{Co}$ where the distribution widths are similar. This again points to different mechanisms for the Kondo screening for $\mathrm{Ni}$, on the one hand, and for $\mathrm{Co}$ and $\mathrm{Fe}$, on the other hand.

(iv) The occupations $n_{d}$ of the impurity level giving rise to the Kondo resonance calculated from $T_{K}$ and $\epsilon_{K}$ by Eq. (6) again follow a Gaussian distribution centered around occupation 1. This distribution is narrower for $\mathrm{Ni}$ than for $\mathrm{Co}$ and Fe. This can be understood by considering that most likely for Ni only a single $d$ level is active while for Fe and Co several $d$ levels must be active. This leads to stronger charge fluctuations and, hence, a larger variation in the $d$-level occupations in the cases of $\mathrm{Co}$ and $\mathrm{Fe}$.

(v) The distribution of the Fano curve amplitudes does not show significant differences between the materials. We find that the square root of the amplitudes is normally distributed. This again can be understood by a normal distribution of the characteristic parameters of the nanocontacts on which the amplitude depends quadratically.

(vi) The plot of the amplitudes against the Kondo temperatures follows a similar (almost linear) trend for the three materials. When divided by the mean values for each material the data lines are perfectly on top of each other, showing a universal scaling behavior of the amplitude with the Kondo temperature.

(vii) There are more asymmetric Fano line shapes in the case of $\mathrm{Ni}$ than for $\mathrm{Co}$ and $\mathrm{Fe}$. The distribution of Fano parameters $q$ again is similar for $\mathrm{Fe}$ and $\mathrm{Co}$ but differs for $\mathrm{Ni}$. Co and $\mathrm{Fe}$ show a clear preference for the diplike $q=0$ line shapes while for
TABLE IV. Summary of the different parameters extracted from the statistical analysis of hundreds of atomic contacts for each of the three materials $\mathrm{Fe}, \mathrm{Co}$, and Ni.

\begin{tabular}{lccc}
\hline \hline & $\mathrm{Fe}$ & $\mathrm{Co}$ & $\mathrm{Ni}$ \\
\hline Mean $T_{K}$ & 90 & 120 & 280 \\
FWHM $\ln \left(T_{K}\right)$ & 0.96 & 0.91 & 0.73 \\
Mean $\epsilon_{K}(\mathrm{mV})$ & -1.0 & 0.6 & 0.3 \\
FWHM $\epsilon_{K}(\mathrm{mV})$ & 14.7 & 16.3 & 25.4 \\
Mean $n_{d}$ & 1.07 & 0.97 & 0.99 \\
FWHM $n_{d}$ & 0.79 & 0.74 & 0.52 \\
Mean $A$ from $\sqrt{A}$ & 0.13 & 0.088 & 0.1 \\
FWHM $\sqrt{(} A)$ & 0.31 & 0.24 & 0.27 \\
\hline \hline
\end{tabular}

Ni the distribution of the Fano parameters is somewhat more uniform.

Table IV shows a summary of the parameters extracted from the statistical analysis of the Fano-Kondo line shapes for the all three materials.

\section{DISCUSSION}

The picture of the Kondo effect in ferromagnetic atomic contacts that emerges from our statistical analysis and theoretical considerations is as follows: The low coordination and disorder of the atoms in the contact region in connection with a higher effective Coulomb repulsion can lead to the localization of a single spin in an individual $d$ level of an atom in the contact region. ${ }^{38}$ Now, due to disorder in the contact region, it can be that this $d$ level couples only very weakly to the spin-polarized $d$ levels on neighboring atoms but instead it has an effective coupling to the basically spin-unpolarized $s$-type conduction channel. In this situation, the Kondo effect can take place and screen the spin in that $d$ level.

In the case of $\mathrm{Ni}$ there is one hole in the $3 d$ shell and, therefore, a spin-1/2 associated with it. If the spin becomes localized in a $d$ level that predominantly couples to the spin-unpolarized $s$-type conduction electrons, this spin can be fully screened. Hence, for $\mathrm{Ni}$ we should have a normal Kondo effect where the full spin-1/2 is screened. In the cases of $\mathrm{Fe}$ and Co the atomic spin is higher due to three and two holes, respectively, in the $3 d$ shell of these atoms. This might explain why the measured Kondo temperatures are higher in the case of $\mathrm{Ni}$ than for $\mathrm{Co}$ and $\mathrm{Fe}$ since the Kondo temperature decreases with increasing spin of the impurity. ${ }^{81,82}$ However, one would then also expect a significantly lower Kondo temperature for Fe than for Co which is not the case.

The scenario that we propose instead for $\mathrm{Co}$ and $\mathrm{Fe}$ is an underscreened Kondo effect where the full atomic spin $S>1 / 2$ is only partially screened by the conduction electrons. More precisely, only the spin-1/2 within the $d$ level that predominantly couples to the $s$-type conduction electrons will be screened while the rest of the spin $S-1 / 2$, which is likely to be localized in $d$ levels that couple more strongly to the spin-polarized $d$ levels on neighboring atoms, remains unscreened. This scenario fits very well with our results: In particular, it explains why the average Kondo temperature is very similar for $\mathrm{Fe}$ and $\mathrm{Co}$ but is considerably higher for $\mathrm{Ni}$ as the underscreened Kondo effect is characterized by a cusplike 
resonance much sharper than the Lorentzian-type resonance of the normal fully screened Kondo effect. An underscreened Kondo effect for $\mathrm{Fe}$ and Co contacts would also explain why the occupations can be calculated from Eq. (6) derived for the single-level Anderson impurity model.

Recent work by Néel et al. ${ }^{37}$ shows how the Kondo temperature increases for a cobalt impurity when it is contacted by an Fe tip compared to the case of using a $\mathrm{Cu}$ tip. These results and their interpretation are in good agreement with the results presented here: differences in the composition and geometries of the junctions lead to different electronic structures of the contact which determines the Kondo screening. By studying a specific system with a well-characterized geometry, Neel et al. can extract the change in hybridization and the corresponding occupation of the $d$ level as the main cause of the observed increase in Kondo temperatures. We study here instead a large variety of possibilities. Changes in the hybridization are probably responsible for the broad distribution of Kondo temperatures in our data, but other parameters, such as the occupancy of the $3 d$ shell, may also change in our system from contact to contact.

Finally, we discuss the connection to a related work by Bork et $a l .{ }^{36}$ with the results presented here: In the work of Bork et al., the Fano-Kondo line shapes of a system consisting of two Co atoms, one on a $\mathrm{Cu}$ surface and the other attached to a Cu STM tip, are recorded while the distance between the two Co atoms is decreased from the tunneling regime to the contact regime. The authors report a splitting of the Fano-Kondo line shapes when the contact regime is entered due to the formation of a spin-singlet state between the two Co atoms.

In some cases, the Fano-Kondo line shapes of the ferromagnetic contacts measured here could also be interpreted as a splitting of the Kondo resonance. For example, the evolution of the Fano line shape of a Co nanocontact being stretched (Fig. 11) possibly shows a small splitting for the curve with the lowest conductance. However, if this specific feature really shows a splitting of the Fano-Kondo resonance, it actually occurs in the opposite direction as for the system studied by Bork et al., i.e., it occurs when the nanocontact is pulled apart and not when the tip atoms are brought together as in the case of Bork et al. In any case, we have not observed very frequently such curves that could be interpreted as splittings, and the focus of this work is a statistical analysis of a large number of different configurations of atomic contacts while the work of Bork et al. focuses on a very specific system. On the other hand, we also would like to point out that conductance oscillations in the spectroscopy at the atomic scale ${ }^{83}$ further complicate the interpretation of these kinds of curves.

Still one might wonder why splitting of the Fano-Kondo line shapes in the atomic contacts studied here are not observed more frequently. The reason might be that indeed the $d$ level on a tip atom giving rise to the Kondo effect just does not couple very well to the $d$ levels of the other tip atom due to the disorder in the contact region. Hence, the splitting is probably very weak compared to the Kondo temperature and, therefore, not observed, as also shown in Ref. 37, where the Kondo resonance remains unsplitted even when the Co adatom is contacted by a ferromagnetic tip. Another possible explanation could be that the weak coupling between the $d$ levels giving rise to the Kondo effect localized on different tip atoms is compensated by the weak spin polarization of the conduction electrons, similarly to the case of a double quantum dot coupled to ferromagnetic leads. ${ }^{84}$

\section{CONCLUSIONS}

Atomic contacts are unique systems that allow us to understand the dramatic consequences of low coordination and disorder on the electronic transport and magnetic properties at the nanoscale. The emergence of the Kondo effect in atomic contacts is a good example of this. ${ }^{38}$ Here we have extended our previous work in several aspects in order to gain new insights into the nature of the Kondo effect in ferromagnetic one-atom contacts: We have presented an exhaustive statistical analysis of the Fano resonances in the spectroscopy of ferromagnetic one-atom contacts. In particular, we have analyzed not only the distribution of Kondo temperatures but also of the resonance energies, amplitudes, and Fano parameters. Such an exhaustive statistical analysis provides us with new information on the nature of the Kondo effect in these systems.

From this analysis, we have, for example, obtained the distribution of $d$-level occupations, and the dependence of the amplitudes of the Fano resonance on the Kondo temperature. We also point out the widths of the parameter distributions as an indicator on the robustness of the Kondo screening in oneatom contacts of the different materials. Additionally, we have further developed the theory in order to explain the observed qualitative differences between $\mathrm{Ni}$, on the one hand, and $\mathrm{Co}$ and $\mathrm{Fe}$, on the other hand. Furthermore, we have devised a simple microscopic model which allows us to understand the occurrence of different Fano line shapes for the same material in terms of variations of the microscopic interactions.

Our statistical analysis shows clear differences in the Kondo characteristics of different ferromagnetic materials, i.e., of $\mathrm{Fe}$ and $\mathrm{Co}$ versus $\mathrm{Ni}$. These differences can be explained by the different valences, leading to different Kondo scenarios. Although this might not be a unique explanation for the observed phenomenology, the reported results seem to fit well with an underscreened Kondo effect for the cases of $\mathrm{Fe}$ and $\mathrm{Co}$ and a standard spin-1/2 Kondo model for the case of nickel.

While the different screening conditions might account for the differences in Kondo temperatures and occupation, the complexity of atomic contacts, especially of transitions metals, where many different channels contribute to the transport, makes it difficult to fully understand the details for each single contact. A simple interpretation for certain parameters as the exact shape of the curves (Fano parameter) or the amplitude of the resonances is difficult to achieve as the spectroscopy also might contain information about other phenomena, such as quantum oscillations, due to interferences between nonresonant conduction channels, inelastic processes as phonon excitations, and so on.

In the recent past, the combination of molecular dynamics simulations and ab initio transport calculations and experiment has proven to be a successful path for understanding the atomic, electronic, and magnetic structures as well as the transport properties of nanocontacts. ${ }^{39,50,59}$ Further work in this direction in combination with more sophisticated manybody techniques capable of describing the Kondo effect, such as the dynamical mean-field theory, ${ }^{60,85}$ should contribute to 
fully understand Kondo physics and the presence of magnetism in these systems and in atomic-size structures in general.

\section{ACKNOWLEDGMENTS}

This work was partly supported by the Spanish MEC (Grants No. FIS2010-21883-C02-01 and No. CONSOLIDER CSD2007-0010) and Comunidad Valenciana (ACOMP/2012/127 and PROMETEO/2012/011). M. R. Calvo aknowledges financial support from the Spanish MEC. We are grateful to Vicent Esteve for technical support and J. I. Fernández-Rossier, J. J. Palacios, and D. Natelson for stimulating scientific discussions.

\section{APPENDIX A: TWO-LEVEL ANDERSON IMPURITY EIGENSTATES}

Here we briefly discuss the many-body eigenstates of the isolated two-level Anderson impurity model and the fluctuations between them that lead to the underscreened Kondo effect as discussed in Sec. III C.

The isolated impurity Hamiltonian $\hat{H}_{I}$ of the 2AIM (9) is easily diagonalized. The empty impurity state is denoted by $\left|d^{0}\right\rangle$ and has energy $E_{0}=0$. For single occupation, the four eigenstates and energies are trivially given by

$$
\left|d^{1} ; i \sigma\right\rangle=d_{i \sigma}^{\dagger}|0\rangle, \quad E_{1}=\left\langle i \sigma\left|\hat{H}_{I}\right| i \sigma\right\rangle=\epsilon .
$$

For double occupation, the eigenstates can be separated into spin-singlet (total impurity spin $S=0$ ) and spin-triplet ( $S=$ 1) states. The latter are given by

$$
\left|d^{2} ; S=1 ; M\right\rangle=|1,2\rangle^{-} \otimes\left\{\begin{array}{ll}
|\uparrow, \uparrow\rangle & (M=1) \\
|\uparrow, \downarrow\rangle^{+} & (M=0) \\
|\downarrow, \downarrow\rangle & (M=-1)
\end{array},\right.
$$

where $M$ is the projection of the total impurity spin onto the spin quantization axis. The triplet states are all degenerate with energy $E_{T}=2 \epsilon+U^{\prime}-J_{H} / 4$. The spin singlet states can be written as

$$
\left|d^{2} ; S=0 ; i j\right\rangle=|i, j\rangle^{+} \otimes|\uparrow, \downarrow\rangle^{-} .
$$

The corresponding eigenenergies are $E_{S}^{12}=2 \epsilon+U^{\prime}+\frac{3}{4} J_{H}$ and $E_{S}^{11}=E_{S}^{22}=2 \epsilon+U$. Typically, $U \approx U^{\prime}+J_{H}$, and, therefore, $E_{S}^{11}=E_{S}^{22}>E_{S}^{12}$. Due to Hund's rule coupling, the triplet states are lower in energy by an amount $\left(E_{S}^{12}-E\right)_{T}=$ $J_{H}$. In Fig. 12 we show the corresponding energy-level diagram for the 2AIM. $U$ and $U^{\prime}$ are assumed to be big enough to prevent triple and full occupation of the impurity. Hence,

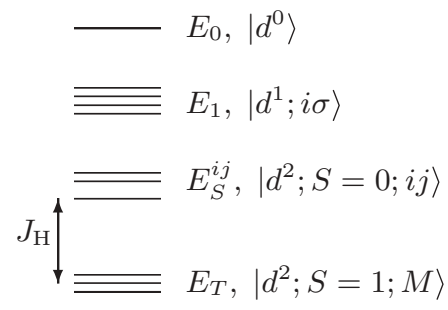

FIG. 12. Schematic energy-level diagram for the (isolated) twolevel impurity described by $H_{\mathrm{I}}$ in Eq. (9). the triplet states comprise the ground-state manifold of the isolated impurity system.

Now in the situation of the underscreened Kondo effect where only one of the impurity levels is coupled to the conduction electron bath, hopping processes between the impurity and the bath can only lead to a spin flip in the impurity level that is coupled to the bath as illustrated in Fig. 3. These spin-flip processes can only lead to fluctuations between states with $M=1$ and $M=0$ and between states with $M=-1$ and $M=0$. For example, $|S=1 ; M=1\rangle=|1 \uparrow, 2 \uparrow\rangle^{(-)} \stackrel{d_{1 \uparrow}}{\longrightarrow}|2 \uparrow\rangle \stackrel{d_{1 \downarrow}^{\dagger}}{\longrightarrow}$ $|1 \downarrow, 2 \uparrow\rangle^{(-)} \stackrel{d_{1 \downarrow}}{\longrightarrow}|2 \uparrow\rangle \stackrel{d_{1 \uparrow}^{\dagger}}{\longrightarrow}|1 \uparrow, 2 \uparrow\rangle^{(-)}$. Hence, the total spin of the impurity is only partially screened by these processes. This is the essence of the underscreened Kondo effect.

\section{APPENDIX B: DERIVATION OF THE FANO FORMULA FOR MODEL CONTACT}

We assume the simplified model of a nanocontact described in Sec. III and shown in Fig. 2. We concentrate on one of the tip atoms of the nanocontact, which, in our model, consists of an (almost) perfectly transmitting $s$ level and the $d$ level where the Kondo effect takes place. Hence, the Greens function (GF) of the tip atom can be written as

$$
\mathbf{G}_{A}(\omega)=\left(\begin{array}{cc}
G_{s} & G_{s d} \\
G_{s d} & G_{d}
\end{array}\right),
$$

where $G_{d}$ is the Green's function of the $d$ level which in the Kondo regime is given by Eq. (2) and, thus, yields a Kondo resonance in the corresponding spectral function as described by Eq. (3) at energy $\epsilon_{K}$, half-width $\Gamma_{K}$, and the quasiparticle weight $z$.

The unperturbed $s$-level GF (i.e., without coupling $V_{s d}$ to the $d$ level) is given by

$$
G_{s}^{0}(\omega)=\frac{1}{\omega-\epsilon_{s}+i \Gamma_{s} / 2} \approx \frac{1}{-\epsilon_{s}+i \Gamma_{s} / 2},
$$

where $\epsilon_{s}$ is the energy of the $s$ level and $\Gamma_{s}=\Gamma_{L, s}+\Gamma_{R, s}$ the width due to coupling to both electrodes. Since the energies we are interested in are on the order of the Kondo scale, $|\omega| \approx \Gamma_{K}$ and $\Gamma_{K} \ll \Gamma_{s}$, we neglect the $\omega$ dependence of $G_{s}^{0}$ in the last step of Eq. (B2).

Due to the coupling $V_{s d}$ of the $s$ level to the $d$ level, the full GF $G_{s}$ of the $s$ level is modified according to

$$
G_{s}(\omega)=G_{s}^{0}(\omega)+G_{s}^{0}(\omega) V_{s d} G_{d}(\omega) V_{s d} G_{s}^{0}(\omega)
$$

The off-diagonal term $G_{s d}$ describes the interference between both channels due to the coupling $V_{s d}$ and is given by

$$
G_{s d}(\omega)=V_{s d} G_{s}^{0} G_{d}(\omega)
$$

As was shown by Meir and Wingreen in their seminal work, ${ }^{75}$ at zero temperature and in linear response, the exact result for the conductance and current through an interacting region reduces to the Landauer result where the transmission function can be calculated from the Caroli formula. ${ }^{86}$ Using the Caroli formula we can calculate the coherent transmission 
via the tip atom as

$$
T(\omega)=\operatorname{Tr}\left[\boldsymbol{\Gamma}_{L} \mathbf{G}_{A}^{\dagger} \boldsymbol{\Gamma}_{R} \mathbf{G}_{A}\right],
$$

where $\boldsymbol{\Gamma}_{L}$ and $\boldsymbol{\Gamma}_{R}$ are the so-called coupling matrices describing the coupling of the tip atom $A$ to the left and right electrodes,

$$
\boldsymbol{\Gamma}_{\alpha}=\left(\begin{array}{cc}
\Gamma_{\alpha, s} & 0 \\
0 & \Gamma_{\alpha, d}
\end{array}\right) \text { with } \alpha \in\{L, R\} .
$$

Hence, we find for the transmission $T(\omega)$ through the tip atom,

$$
T(\omega)=T_{s}(\omega)+T_{d}(\omega)+T_{s d}(\omega),
$$

where $T_{s}$ is the direct transmission through the $s$ channel and $T_{d}$ the corresponding one through the $d$ channel while $T_{s d}$ describes the transmission involving hopping processes between the $s$ and $d$ channels,

$$
\begin{gathered}
T_{s}(\omega)=2 \pi \frac{\Gamma_{L, s} \Gamma_{R, s}}{\Gamma_{s}} \rho_{s}(\omega), \\
T_{d}(\omega)=2 \pi \frac{\Gamma_{L, d} \Gamma_{R, d}}{\Gamma_{d}} \rho_{d}(\omega), \\
T_{s d}(\omega)=\left(\Gamma_{L, s} \Gamma_{R, d}+\Gamma_{L, d} \Gamma_{R, s}\right)\left|G_{s d}\right|^{2} .
\end{gathered}
$$

In order to calculate the contribution of the $s$ channel to the total transmission, we need to know the spectral density of the $s$ level, which is given by the imaginary part of the $s$-level GF given by Eq. (B3),

$$
\rho_{s}(\omega)=\underbrace{-\frac{1}{\pi} \operatorname{Im}\left[G_{s}^{0}(\omega)\right]}_{\rho_{s}^{0}(\omega)} \underbrace{-\frac{V_{s d}^{2}}{\pi} \operatorname{Im}\left[\left(G_{s}^{0}(\omega)\right)^{2} G_{d}(\omega)\right]}_{\delta \rho_{s}(\omega)}
$$

where $\rho_{s}^{0}$ is the spectral function of the unperturbed $s$ level, which in our model is constant, $\rho_{s}^{0}=\Gamma_{s} / 2 \pi\left(\Gamma_{s}^{2} / 4+\epsilon_{s}^{2}\right)$. $\delta \rho_{s}(\omega)$ is the change in the spectral density due to the coupling to the $d$ level with the Kondo peak. For the latter we find

$$
\begin{aligned}
\delta \rho_{s}= & -\frac{V_{s d}^{2}}{\pi}\left\{\operatorname{Im}\left[\left(G_{s}^{0}\right)^{2}\right] \operatorname{Re}\left[G_{d}\right]+\operatorname{Re}\left[\left(G_{s}^{0}\right)^{2}\right] \operatorname{Im}\left[G_{d}\right]\right\} \\
= & -\frac{V_{s d}^{2}}{\pi}\left\{2 \operatorname{Re}\left[G_{s}^{0}\right] \operatorname{Im}\left[G_{s}^{0}\right] \operatorname{Re}\left[G_{d}\right]\right. \\
& \left.+\left(\operatorname{Re}\left[G_{s}^{0}\right]^{2}-\operatorname{Im}\left[G_{s}^{0}\right]^{2}\right) \operatorname{Im}\left[G_{d}\right]\right\} .
\end{aligned}
$$

The real and imaginary part of $G_{d}$ are given by

$$
\begin{gathered}
\operatorname{Re} G_{d}(\omega)=\frac{z\left(\omega-\epsilon_{K}\right)}{\left(\omega-\epsilon_{K}\right)^{2}+\Gamma_{K}^{2}}=\frac{z}{\Gamma_{K}} \frac{x}{x^{2}+1}, \\
-\operatorname{Im} G_{d}(\omega)=\frac{z \Gamma_{K}}{\left(\omega-\epsilon_{K}\right)^{2}+\Gamma_{K}^{2}}=\frac{z}{\Gamma_{K}} \frac{1}{x^{2}+1},
\end{gathered}
$$

where we have defined $x \equiv\left(\omega-\epsilon_{K}\right) / \Gamma_{K}$. Plugging this into Eq. (B12), we find

$$
\begin{aligned}
\delta \rho_{s}= & -\frac{z V_{s d}^{2}}{\pi \Gamma_{K}} \frac{1}{x^{2}+1}\left\{2 x \operatorname{Re} G_{s}^{0} \operatorname{Im} G_{s}^{0}-\left[\operatorname{Re} G_{s}^{0}\right]^{2}\right. \\
& \left.+\left[\operatorname{Im} G_{s}^{0}\right]^{2}\right\} .
\end{aligned}
$$

We now define the ratio $q$ between the real and imaginary parts of $G_{s}^{0}$,

$$
q \equiv-\frac{\operatorname{Re} G_{s}^{0}}{\operatorname{Im} G_{s}^{0}} \approx-\frac{2 \epsilon_{s}}{\Gamma_{s}}
$$

With this we find for the expression in curly brackets in Eq. (B15)

$$
\begin{aligned}
2 x & \operatorname{Re} G_{s}^{0} \operatorname{Im} G_{s}^{0}-\left[\operatorname{Re} G_{s}^{0}\right]^{2}+\left[\operatorname{Im} G_{s}^{0}\right]^{2} \\
& =\left[\operatorname{Im} G_{s}^{0}\right]^{2}\left\{2 x \frac{\operatorname{Re} G_{s}^{0}}{\operatorname{Im} G_{s}^{0}}-\left[\frac{\operatorname{Re} G_{s}^{0}}{\operatorname{Im} G_{s}^{0}}\right]^{2}+1\right\} \\
& \approx \frac{4}{\Gamma_{s}^{2}} \frac{1}{\left(1+q^{2}\right)^{2}}\left\{-2 q x-q^{2}+1\right\} \\
& =-4 \frac{(x+q)^{2}-\left(x^{2}+1\right)}{\Gamma_{s}^{2}\left(1+q^{2}\right)^{2}} .
\end{aligned}
$$

Hence, we obtain for the change $\delta \rho_{s}$ in the spectral density of the $s$ level due to the coupling to the $d$ level,

$$
\delta \rho_{s}(\omega)=\frac{4 z V_{s d}^{2}}{\pi \Gamma_{K} \Gamma_{s}^{2}} \frac{(x+q)^{2}-\left(x^{2}+1\right)}{\left(x^{2}+1\right)\left(1+q^{2}\right)^{2}} .
$$

Summing up, we find for the $s$-channel contribution to the transmission (14)

$$
\begin{aligned}
T_{s}(\omega) & =T_{s}^{0}+2 \pi \frac{\Gamma_{L, s} \Gamma_{R, s}}{\Gamma_{s}} \delta \rho_{s}(\omega) \\
& =T_{s}^{0}\left[1+\frac{2 z V_{s d}^{2}}{\Gamma_{s} \Gamma_{K}} \frac{(x+q)^{2}-\left(x^{2}+1\right)}{\left(x^{2}+1\right)\left(1+q^{2}\right)}\right],
\end{aligned}
$$

where $T_{s}^{0}=2 \pi \rho_{s}^{0}\left(\Gamma_{L, s} \Gamma_{R, s}\right) / \Gamma_{s}$ is the transmission via the unperturbed $s$ channel.

For the $d$-channel contribution to the total transmission we find, according to Eqs. (B7) and (3),

$$
T_{d}(\omega)=4 \frac{\Gamma_{L, d} \Gamma_{R, d}}{\Gamma_{d}^{2}} \frac{1}{x^{2}+1} .
$$

Finally, for the $s d$-coupling contribution to the transmission we need to calculate the absolute square of the off-diagonal matrix element $G_{s d}$ of the atomic GF,

$$
\left|G_{s d}(\omega)\right|^{2}=V_{s d}^{2}\left|G_{s}^{0}\right|^{2}\left|G_{d}(\omega)\right|^{2}=\frac{V_{s d}^{2} z^{2}}{\Gamma_{K}^{2}\left(1+x^{2}\right)} .
$$

Hence, we find

$$
T_{s d}(\omega)=T_{s}^{0} \frac{z^{2} V_{s d}^{2}}{\Gamma_{K}^{2}}\left(\frac{\Gamma_{R, d}}{\Gamma_{R, s}}+\frac{\Gamma_{L, d}}{\Gamma_{L, s}}\right) \frac{1}{1+x^{2}} .
$$


${ }^{1}$ W. J. de Haas, J. de Boer, and G. J. van den Berg, Physica (Utrecht) 1, 1115 (1934).

${ }^{2}$ J. Kondo, Prog. Theor. Phys. 32, 37 (1964).

${ }^{3}$ D. Goldhaber-Gordon, H. Shtrikman, D. Mahalu, D. AlbuschMadger, U. Meirav, and M. A. Kastner, Nature 391, 156 (1998).

${ }^{4}$ S. M. Cronenwett, T. H. Oosterkamp, and L. P. Kouwenhoven, Science 281, 540 (1998).

${ }^{5}$ M. A. Kastner, Rev. Mod. Phys. 64, 849 (1992).

${ }^{6}$ A. C. Hewson, The Kondo Problem to Heavy Fermions (Cambridge University, Cambridge, 1993).

${ }^{7}$ A. A. Abrikosov, Physics 2, 5 (1965).

${ }^{8}$ H. Suhl, Physics 2, 39 (1965).

${ }^{9}$ Y. Nagaoka, Phys. Rev. 138, A1112 (1965).

${ }^{10}$ T. Rejec and Y. Meir, Nature 442, 900 (2006).

${ }^{11}$ I. K. Yanson, V. V. Fisun, R. Hesper, A. V. Khotkevich, J. M. Krans, J. A. Mydosh, and J. M. van Ruitenbeek, Phys. Rev. Lett. 74, 302 (1995).

${ }^{12}$ W. Liang, M. P. Shores, M. Bockrath, J. R. Long, and H. Park, Nature 417, 725 (2002).

${ }^{13}$ J. Park, A. N. Pasupathy, J. I. Goldsmith, C. Chang, Y. Yaish, J. R. Petta, M. Rinkoski, J. P. Sethna, H. D. Abruña, P. L. McEuen, and D. C. Ralph, Nature 417, 722 (2002).

${ }^{14}$ L. H. Yu and D. Natelson, Nano Lett. 4, 79 (2003).

${ }^{15}$ J. Nygard, D. H. Cobden, and P. E. Lindelof, Nature 408, 342 (2000).

${ }^{16}$ J. Kim, J.-R. Kim, Jeong-O. Lee, J. W. Park, H. M. So, N. Kim, K. Kang, K.-H. Yoo, and J.-J. Kim, Phys. Rev. Lett. 90, 166403 (2003).

${ }^{17}$ V. Mandhavan, W. Chen, T. Jamneala, M. F. Crommie, and N. S. Wingreen, Science 280, 567 (1998).

${ }^{18}$ J. Li, W. D. Schneider, R. Berndt, and B. Delley, Phys. Rev. Lett. 80, 2893 (1998).

${ }^{19}$ A. F. Otte, M. Ternes, K. von Bergmann, S. Loth, H. Brune, C. P. Lutz, C. F. Hirjibehedin, and A. J. Heinrich, Nat. Phys. 4, 847 (2008).

${ }^{20}$ M. Ternes, A. J. Heinrich, and W.-D. Schneider, J. Phys.: Condens. Matter 21, 053001 (2009).

${ }^{21}$ V. Madhavan, W. Chen, T. Jamneala, M. F. Crommie, and N. S. Wingreen, Phys. Rev. B 64, 165412 (2001).

${ }^{22}$ N. Knorr, M. A. Schneider, L. Diekhöner, P. Wahl, and K. Kern, Phys. Rev. Lett. 88, 096804 (2002).

${ }^{23}$ P. Wahl, L. Diekhöner, M. A. Schneider, L. Vitali, G. Wittich, and K. Kern, Phys. Rev. Lett. 93, 176603 (2004).

${ }^{24}$ K. Nagaoka, T. Jamneala, M. Grobis, and M. F. Crommie, Phys. Rev. Lett. 88, 077205 (2002).

${ }^{25}$ T. Jamneala, V. Madhavan, W. Chen, and M. F. Crommie, Phys. Rev. B 61, 9990 (2000).

${ }^{26}$ U. Fano, Phys. Rev. 124, 1866 (1961).

${ }^{27}$ J. Göres, D. Goldhaber-Gordon, S. Heemeyer, M. A. Kastner, H. Shtrikman, D. Mahalu, and U. Meirav, Phys. Rev. B 62, 2188 (2000).

${ }^{28}$ M. Sato, H. Aikawa, K. Kobayashi, S. Katsumoto, and Y. Iye, Phys. Rev. Lett. 95, 066801 (2005).

${ }^{29}$ N. Néel, J. Kröger, L. Limot, K. Palotas, W. A. Hofer, and R. Berndt, Phys. Rev. Lett. 98, 016801 (2007).

${ }^{30}$ L. Vitali, R. Ohmann, S. Stepanow, P. Gambardella, K. Tao, R. Huang, V. S. Stepanyuk, P. Bruno, and K. Kern, Phys. Rev. Lett. 101, 216802 (2008).

${ }^{31}$ N. Néel, J. Kröger, R. Berndt, T. O. Wehling, A. I. Lichtenstein, and M. I. Katsnelson, Phys. Rev. Lett. 101, 266803 (2008).
${ }^{32}$ J. Kröger, N. Néel, and L. Limot, J. Phys.: Condens. Matter 20, 223001 (2008).

${ }^{33}$ N. Néel, J. Kröger, R. Berndt, and E. Pehlke, Phys. Rev. B 78, 233402 (2008).

${ }^{34}$ N. Néel, J. Kröger, and R. Berndt, Phys. Rev. Lett. 102, 086805 (2009).

${ }^{35}$ J. Kröger, A. Sperl, N. Néel, and R. Berndt, J. Scanning Probe Microsc. 4, 49 (2009).

${ }^{36}$ J. Bork, Y. H. Zhang, L. Diekhöner, L. Borda, P. Simon, J. Kroha, P. Wahl, and K. Kern, Nat. Phys. 7, 901 (2011).

${ }^{37}$ N. Néel, J. Kröger, and R. Berndt, Phys. Rev. B 82, 233401 (2010).

${ }^{38}$ M. R. Calvo, J. Fernández-Rossier, J. J. Palacios, D. Jacob, D. Natelson, and C. Untiedt, Nature 458, 1150 (2009).

${ }^{39}$ C. Sabater, C. Untiedt, J. J. Palacios, and M. J. Caturla, Phys. Rev. Lett. 108, 205502 (2012).

${ }^{40}$ N. Agraï, A. Levy-Yeyati, and J. M. van Ruitenbeek, Phys. Rep. 377, 81 (2003).

${ }^{41}$ T. A. Costi, Phys. Rev. Lett. 85, 1504 (2000).

${ }^{42}$ A. Kogan, S. Amasha, D. Goldhaber-Gordon, G. Granger, M. A. Kastner, and H. Shtrikman, Phys. Rev. Lett. 93, 166602 (2004).

${ }^{43}$ C. H. L. Quay, J. Cumings, S. J. Gamble, R. de Picciotto, H. Kataura, and D. Goldhaber-Gordon, Phys. Rev. B 76, 245311 (2007).

${ }^{44}$ J. Martinek, Y. Utsumi, H. Imamura, J. Barnaś, S. Maekawa, J. König, and G. Schön, Phys. Rev. Lett. 91, 127203 (2003).

${ }^{45}$ M.-S. Choi, D. Sánchez, and R. López, Phys. Rev. Lett. 92, 056601 (2004).

${ }^{46}$ J. R. Hauptmann, J. Paaske, and P. Lindelof, Nat. Phys. 4, 373 (2008).

${ }^{47}$ A. N. Pasupathy, R. C. Bialczak, J. Martinek, J. E. Grose, L. A. K. Donev, P. L. McEuen, and D. C. Ralph, Science 306, 86 (2004).

${ }^{48}$ E. Scheer, N. Agraï, J. C. Cuevas, A. Levy-Yeyati, B. Ludoph, A. Martin-Rodero, G. Rubio-Bollinger, J. M. van Ruitenbeek, and C. Urbina, Nature 394, 154 (1998).

${ }^{49}$ J. C. Cuevas, A. Levy Yeyati, A. Martin-Rodero, G. Rubio Bollinger, C. Untiedt, and N. Agraï, Phys. Rev. Lett. 81, 2990 (1998).

${ }^{50}$ C. Untiedt, M. J. Caturla, M. R. Calvo, J. J. Palacios, R. C. Segers, and J. M. van Ruitenbeek, Phys. Rev. Lett. 98, 206801 (2007).

${ }^{51}$ C. Untiedt, D. M. T. Dekker, D. Djukic, and J. M. van Ruitenbeek, Phys. Rev. B 69, 081401 (2004).

${ }^{52}$ B. Ludoph and J. M. van Ruitenbeek, Phys. Rev. B 61, 2273 (2000).

${ }^{53}$ D. Jacob, J. Fernandez-Rossier, and J. J. Palacios, Phys. Rev. B 71, 220403(R) (2005).

${ }^{54}$ A. Smogunov, A. Dal Corso, and E. Tosatti, Phys. Rev. B 73, 075418 (2006).

${ }^{55}$ A. Bagrets, N. Papanikolaou, and I. Mertig, Phys. Rev. B 75, 235448 (2007).

${ }^{56}$ M. Häfner, J. K. Viljas, D. Frustaglia, F. Pauly, M. Dreher, P. Nielaba, and J. C. Cuevas, Phys. Rev. B 77, 104409 (2008).

${ }^{57}$ P. W. Anderson, Phys. Rev. 124, 41 (1961).

${ }^{58}$ F. Pauly, M. Dreher, J. K. Viljas, M. Häfner, J. C. Cuevas, and P. Nielaba, Phys. Rev. B 74, 235106 (2006).

${ }^{59}$ M. R. Calvo, M. J. Caturla, D. Jacob, C. Untiedt, and J. J. Palacios, IEEE Trans. Nanotechnol. 7, 165 (2008).

${ }^{60}$ D. Jacob, K. Haule, and G. Kotliar, Phys. Rev. B 82, 195115 (2010).

${ }^{61}$ P. Nozieres, J. Low Temp. Phys. 17, 31 (1974).

${ }^{62}$ P. Noziéres and A. Blandin, J. Phys. 41, 193 (1980).

${ }^{63}$ N. B. Perkins, J. R. Iglesias, M. D. Núñez-Regueiro, and B. Coqblin, EPL 79, 57006 (2007). 
${ }^{64}$ P. Coleman and C. Pépin, Phys. Rev. B 68, 220405 (2003).

${ }^{65}$ P. Coleman and I. Paul, Phys. Rev. B 71, 035111 (2005).

${ }^{66}$ W. Koller, A. C. Hewson, and D. Meyer, Phys. Rev. B 72, 045117 (2005).

${ }^{67}$ P. Gentile, L. De Leo, M. Fabrizio, and E. Tosatti, EPL 87, 27014 (2009).

${ }^{68}$ P. Lucignano, R. Mazzarello, A. Smogunov, M. Fabrizio, and E. Tosatti, Nat. Mater. 8, 563 (2009).

${ }^{69}$ A. Posazhennikova, B. Bayani, and P. Coleman, Phys. Rev. B 75, 245329 (2007).

${ }^{70}$ D. E. Logan, C. J. Wright, and M. R. Galpin, Phys. Rev. B 80, 125117 (2009).

${ }^{71}$ A. Posazhennikova and P. Coleman, Phys. Rev. Lett. 94, 036802 (2005).

${ }^{72}$ P. Mehta, N. Andrei, P. Coleman, L. Borda, and G. Zarand, Phys. Rev. B 72, 014430 (2005).

${ }^{73}$ W. Koller, A. C. Hewson, and D. Meyer, Phys. Rev. B 72, 045117 (2005).

${ }^{74}$ R. Zitko, Phys. Rev. B 81, 115316 (2010).
${ }^{75}$ Y. Meir and N. S. Wingreen, Phys. Rev. Lett. 68, 2512 (1992).

${ }^{76}$ S.-F. Shi, K. I. Bolotin, F. Kuemmeth, and D. C. Ralph, Phys. Rev. B 76, 184438 (2007).

${ }^{77}$ K. Sekiguchi, A. Yamaguchi, H. Miyajima, A. Hirohata, and S. Usui, Phys. Rev. B 78, 224418 (2008).

${ }^{78}$ K. Gloos, Low Temp. Phys. 35, 935 (2009).

${ }^{79}$ K. Gloos and E. Tuuli, arXiv:1109.3774 (2011).

${ }^{80}$ M. D. Daybell and W. A. Steyert, Rev. Mod. Phys. 40, 380 (1968).

${ }^{81}$ E. C. Goldberg and F. Flores, Phys. Rev. B 77, 125121 (2008).

${ }^{82}$ A. H. Nevidomskyy and P. Coleman, Phys. Rev. Lett. 103, 147205 (2009).

${ }^{83}$ C. Untiedt, G. R. Bollinger, S. Vieira, and N. Agraï, Phys. Rev. B 62, 9962 (2000).

${ }^{84}$ R. Zitko, J. S. Lim, R. Lopez, J. Martinek, and P. Simon, Phys. Rev. Lett. 108, 166605 (2012).

${ }^{85}$ D. Jacob, K. Haule, and G. Kotliar, Phys. Rev. Lett. 103, 016803 (2009).

${ }^{86}$ C. Caroli, R. Combescot, and P. Dederichs, J. Phys. C 4, 916 (1971). 